Ocean Science, 1, 45-79, 2005

www.ocean-science.net/os/1/45/

SRef-ID: 1812-0792/os/2005-1-45

European Geosciences Union

\title{
Formulation of an ocean model for global climate simulations
}

\author{
S. M. Griffies ${ }^{1}$, A. Gnanadesikan ${ }^{1}$, K. W. Dixon ${ }^{1}$, J. P. Dunne ${ }^{1}$, R. Gerdes $^{2}$, M. J. Harrison ${ }^{1}$, A. Rosati ${ }^{1}$, J. L. Russell ${ }^{3}$, \\ B. L. Samuels ${ }^{1}$, M. J. Spelman ${ }^{1}$, M. Winton ${ }^{1}$, and R. Zhang ${ }^{3}$ \\ ${ }^{1}$ NOAA Geophysical Fluid Dynamics Laboratory, Princeton, USA \\ ${ }^{2}$ Alfred-Wegener-Institut für Polar- und Meeresforschung, Bremerhaven, Germany \\ ${ }^{3}$ Program in Atmospheric and Oceanic Sciences, Princeton, USA
}

Received: 4 April 2005 - Published in Ocean Science Discussions: 20 May 2005

Revised: 3 August 2005 - Accepted: 30 August 2005 - Published: 12 September 2005

\begin{abstract}
This paper summarizes the formulation of the ocean component to the Geophysical Fluid Dynamics Laboratory's (GFDL) climate model used for the 4th IPCC Assessment (AR4) of global climate change. In particular, it reviews the numerical schemes and physical parameterizations that make up an ocean climate model and how these schemes are pieced together for use in a state-of-the-art climate model. Features of the model described here include the following: (1) tripolar grid to resolve the Arctic Ocean without polar filtering, (2) partial bottom step representation of topography to better represent topographically influenced advective and wave processes, (3) more accurate equation of state, (4) three-dimensional flux limited tracer advection to reduce overshoots and undershoots, (5) incorporation of regional climatological variability in shortwave penetration, (6) neutral physics parameterization for representation of the pathways of tracer transport, (7) staggered time stepping for tracer conservation and numerical efficiency, (8) anisotropic horizontal viscosities for representation of equatorial currents, (9) parameterization of exchange with marginal seas, (10) incorporation of a free surface that accomodates a dynamic ice model and wave propagation, (11) transport of water across the ocean free surface to eliminate unphysical "virtual tracer flux" methods, (12) parameterization of tidal mixing on continental shelves. We also present preliminary analyses of two particularly important sensitivities isolated during the development process, namely the details of how parameterized subgridscale eddies transport momentum and tracers.
\end{abstract}

Correspondence to: S. M. Griffies

(stephen.griffies@noaa.gov)

\section{Introduction}

The purpose of this paper is to detail the formulation of the ocean model developed by scientists and engineers at NOAA's Geophysical Fluid Dynamics Laboratory (GFDL) for use in our latest global coupled climate model. In particular, the focus is on the numerical algorithms and physical parameterizations which form the fundamentals of the ocean model component. Some of this paper takes the form of a review. We hope that this presentation is useful for readers aiming to understand what is involved with constructing global models. We also highlight some novel scientific issues related to sensitivity of the climate model simulation to (1) the use of real water fluxes rather than virtual tracer fluxes, including the treatment of river runoff and exchange with semi-enclosed basins, (2) the algorithm for time stepping the model equations, (3) sensitivity of the extra-tropical circulation to horizontal viscosity, and (4) treatment of the tracer transport associated with mesoscale eddies (i.e. neutral physics parameterizations).

\subsection{Documentation of ocean climate models}

Many issues forming the fundamental elements of ocean climate models are often briefly mentioned in papers primarily concerned with describing simulation characteristics, or they may be relegated to non-peer reviewed technical reports. Such discussions often leave the reader with little intellectual or practical appreciation for the difficult and critical choices made during model development. Our goal here is to partially remedy this situation by focusing on numerical and physical details of the most recent GFDL ocean climate model. In so doing, we expose some of the inner workings of the model and attempt to rationalize choices made during

(C) 2005 Author(s). This work is licensed under a Creative Commons License. 
the development process. Along the way, we identify places where further research and development may be warranted.

This paper is written on the premise that the evolution of climate science is facilitated by a candid peer-reviewed discussion of the interdependent and nontrivial choices that developers make in constructing global climate models. The importance of such discussions has grown during the past decade as the models are used for an increasing variety of applications, many of which, such as climate change projections, garner intense scrutiny from non-scientific communities. Additionally, full disclosure is necessary for modelers to reproduce each other's results, and thus to enhance the scientific robustness of climate modelling.

We admittedly fall short of fully realizing our goals in writing this paper. First, choices were made to balance conciseness with completeness. A substantially longer paper with more thorough analysis of sensitivity experiments and comparisons to observations is required to satisfy the completeness goal. Such analyses form the basis of a number of separate studies described below. Second, we are limited by focusing on one particular climate model, that from GFDL contributing to AR4. Comparisons with other models, such as the earlier GFDL climate models, go beyond the scope of this study.

The evaluation of a global climate model requires years of research by many scientists. It is therefore impossible for any single document to do justice to a particular model's scientific integrity. Instead, full scientific judgement requires a suite of studies from many perspectives. Given the limitations of the present work, we remain hopeful that this paper serves as a step towards full disclosure of the rationale forming the basis for a particular ocean climate model. We believe such provides the climate science community with a useful resource for understanding both how to reproduce elements of what we have done, and to expose areas where further research and development is warranted.

\subsection{Comments on ocean climate model development}

One of the first global coupled climate models was that of Manabe and Bryan (1969). Their model used an early version of the GFDL geopotential vertical coordinate ocean model based on the work of Bryan and Cox (1967) and Bryan (1969b), with Bryan (1969a) documenting algorithms used in this model. It is notable that such $z$-models, which typically employ the hydrostatic and Boussinesq approximations, still comprise the vast majority of ocean models used for climate simulations (see Griffies et al., 2000a, for a review). In particular, all versions of the GFDL coupled climate models to date have employed this class of ocean model.

In most $z$-models used for climate studies through the early 1990's, the ocean primitive equations were discretized using spherical coordinates for the lateral directions, with vertical positions at fixed depths for all latitude and longi- tude points, and with grid cells of time independent volumes. Additionally, physical processes such as ocean tracer transport were aligned according to this grid. Since the middle 1990 's, there have been fundamental advances to this older model formulation that significantly enhance the physical integrity of $z$-model simulations (see Griffies et al., 2000a, for a review). It is therefore important to include these advances in the ocean climate models used for realistic climate simulations.

There are two main ways in which climate modellers seek realism in their simulations. First, individual processes should be represented or parameterized to the best of our understanding. The present paper is directly aimed at articulating our choices for physical processes, with additional discussions of numerical methods. Second, the simulation should behave like the observed climate. This paper is not directly related to this goal, nor do we presume that any single paper is sufficient. Instead, we refer the reader to a growing suite of studies that evaluate the climate model simulation as compared to observations and to other models. The following list provides a sample of manuscripts, available from GFDL, that detail various studies. These studies indicate that the recent GFDL model produces climate simulations that are realistic, and in particular are superior to the older results from the previous GFDL model documented by Delworth et al. (2002).

- Delworth et al. (2005): This paper presents the climate model and illustrates some of its characteristic simulation properties. Notably, the model does not employ artificial flux adjustments used in the previous generation of GFDL climate models such as that documented by Delworth et al. (2002).

- Wittenberg et al. (2005): This paper focuses on the tropical simulation in the climate model, with particular focus on the El Niño Southern Oscillation.

- Stouffer et al. (2005): This paper discusses idealized response in the climate model due to changes in greenhouse gas concentrations.

- Gnanadesikan et al. (2005a): This paper presents a preliminary analysis of the ocean simulations within the coupled climate model, and describes biases and potential origins of these biases.

- Russell et al. (2005) ${ }^{1}$ : This paper compares the Southern Ocean simulations in the control experiments from a suite of IPCC climate models, including the GFDL model.

\footnotetext{
${ }^{1}$ Russell, J., Stouffer, R., and Dixon, K.: Intercomparison of the Southern Ocean Circulations in the IPCC Coupled Model Control Simulations, Journal of Climate, submitted, 2005.
} 


\subsection{Models discussed in this paper}

Throughout this paper, we focus on two versions of the latest GFDL coupled climate model: CM2.0 and CM2.1. These versions have corresponding ocean model versions denoted OM3.0 and OM3.1. The model versions differ in the following ways.

The first difference is in the atmospheric component. CM2.0 uses a B-grid dynamical core documented by Anderson et al. (2005). CM2.1 uses the finite volume core of Lin (2004). Both atmospheric models use similar physical parameterizations. As discussed in Delworth et al. (2005), the mid-latitude storm tracks in both hemispheres are shifted poleward in CM2.1 relative to CM2.0, with the largest shift (order $3-4^{\circ}$ ) in the Southern Hemisphere. This wind shift causes a nontrivial change in the ocean circulation in both hemispheres that significantly reduces middle to high latitude ocean biases in CM2.1 relative to CM2.0 (see Delworth et al., 2005; Gnanadesikan et al., 2005a, for full discussion).

The second difference is in the ocean model, with motivation for these changes provided in this paper. These differences are the following.

- OM3.0 uses a centered in time discretization for the time tendency (i.e. leap frog for the inviscid terms), whereas OM3.1 uses a staggered time stepping scheme.

- OM3.1 uses a constant neutral diffusivity of $600 \mathrm{~m}^{2} \mathrm{~s}^{-1}$. OM3.0 uses a nonconstant diffusivity equal to the skew diffusivity, and this diffusivity is generally less than the $600 \mathrm{~m}^{2} \mathrm{~s}^{-1}$ used in OM3.1.

- OM3.0 uses five times larger background horizontal viscosity poleward of $20^{\circ}$ than OM3.1.

\subsection{Organization of this paper}

This paper consists of two main sections along with an appendix. In Sect. 2, we summarize how various methods and parameterizations documented in other studies have been incorporated into our ocean climate model. This section represents a review of certain elements of ocean climate modelling that have been found to be critical in the construction of our model. Section 3 focuses on experiences and methods that are novel to this work. In particular, Sect. 3.1 explores the issues involved with switching from the commonly used virtual tracer fluxes to real water forcing. We then discuss time stepping algorithms in Sect. 3.2, where we highlight the utility of a time staggered scheme for ocean climate modelling. Neutral physics parameterizations are described in Sect. 3.3, where we note the reasons for changing the subgrid scale (SGS) parameters mentioned above. Horizontal friction is presented in Sect. 3.4, where we show the rather large sensitivity of the simulation to the reduction in extra-tropical viscosity. Section 3.5 details our method for exchanging water mass properties between the open ocean and semi-enclosed basins, and Sect. 3.6 presents our approach for inserting river runoff into the ocean model. Both topics require some novel considerations due to our use of real water fluxes rather than virtual tracer fluxes. We close the paper in Sect. 4 with general comments about ocean climate model development. An appendix of model equations is given to support many discussions in the main text.

\section{Elements of the ocean model based on other work}

When constructing an ocean climate model, it is necessary to choose from amongst a multitude of possible numerical and physical methods. We present here a compendium of model features that have been documented in other studies which are essential elements to our ocean climate model. The main aim here is to motivate choices.

Our discussion of choices made in this section is brief. We do not, for example, provide illustrations of the sensitivity of our model to every alternative choice. For example, when describing the model's tripolar gridding of the sphere in Sect. 2.1, we argue for its benefits over spherical grids, yet do so without providing a direct comparison of simulations with and without the tripolar grid. Instead, this choice, and many others, are based on the judgement and experience of the developers as well as recommendations made in the published literature. Thorough model sensitivity experiments and analysis to justify every model choice are precluded by human, computer, and time limitations. Nonetheless, these choices are acknowledged, as they are important for defining the model fundamentals as well as its simulation.

\subsection{Tripolar grid}

It has become common during the past decade for global ocean models to remove the Arctic Ocean's spherical coordinate singularity via a coordinate transformation to a nonspherical set of generalized orthogonal coordinates. In these models, the coordinate singularity is hidden over land. Removing the coordinate singularity allows modellers to eliminate polar filtering commonly used in spherical coordinate global models (Bryan et al., 1975; Pacanowski and Griffies, 1999), as well as to remove the island present at the North Pole handicapping cross polar flow.

Polar filtering was commonly used in spherical coordinate global models to increase the length of the model's time step. It did so by filtering small scale features poleward of a chosen latitude. Unfortunately, filtering adds an unphysical, and often nontrivial, term to the prognostic equations. An additional problem arises from land-sea boundaries that split the filtered latitudes into distinct sectors which preclude an efficient decomposition of model fields into Fourier modes. As a result, ocean simulations can become noisy in polar filtering regions, even though the goal of filtering is to smooth the fields by removing small scales. 
There is a relatively mature literature detailing methods for removing the spherical coordinate singularity from the Arctic Ocean. Papers by Deleersnijder et al. (1993), Coward et al. (1994), Eby and Holloway (1994), Smith et al. (1995), Murray (1996), Madec and Imbard (1996), Bentsen et al. (1999), Murray and Reason (2002), Marsland et al. (2003), and Roberts et al. (2005) provide various options, present simulation comparisons, and detail various coordinate choices. Our conclusion from this literature is that generalized orthogonal grids are of use for our global modelling efforts.

Given the above motivation to have a non-spherical grid in the Arctic Ocean, it is notable that a spherical grid is quite useful for the remainder of the World Ocean. For example, grid refinement for better representation of the equatorial wave guide is straightforward in a spherical grid. Furthermore, aligning the grid with constant latitude and longitude circles outside the Arctic simplifies the analysis of zonal and meridional transports of properties such as mass and heat.

For the above reasons, in the design of OM3 a primary aim was to remove the spherical coordinate singularity in the Arctic Ocean without affecting the region south of the Arctic. The tripolar grid of Murray (1996) (see his Fig. 7) has proven to be an effective means to achieve this goal, as well as to more evenly distribute grid points within the Arctic region than available with a spherical grid.

This tripolar grid is a composite of two grids, with a familiar spherical, or latitude-longitude, grid south of $65^{\circ} \mathrm{N}$. In the Arctic north of $65^{\circ} \mathrm{N}$, the grid switches to a bipolar region with coordinate singularities over Siberia and Canada. Because all coordinate singularities are hidden inside land masses, they play a negligible role in setting the model time step. The switch between spherical and bipolar Arctic introduces a discontinuity in the derivative of the meridional grid spacing at $65^{\circ} \mathrm{N}$. We have found no sign of this discontinuity in the fields (e.g. tracers, velocity, surface height) simulated on this grid. ${ }^{2}$ Both the ocean and sea ice models in CM2 use the same grid.

Figure 1 presents the land-sea mask within the bipolar Arctic region, along with a few grid lines. The grid is logically rectangular, and so there is nothing special in the model algorithms/code that needs to know about the transition between spherical and bipolar. Additionally, as revealed by Fig. 7 of Murray (1996), the coordinate lines transition into the Arctic in a way that facilitates sensible diagnostics, such as transport streamfunctions and poleward heat transport, when summing along constant $i$-lines. This property greatly simplifies the analysis of model output.

Without polar filtering in a spherical coordinate version of OM3, a linear stability analysis, assuming maximum $2 \mathrm{~m} \mathrm{~s}^{-1}$ wave speed, indicates that a time step would need to be smaller than roughly 800 s using the time stepping scheme

\footnotetext{
${ }^{2}$ A similar grid, with smoother transition to a bipolar Arctic, has been implemented by Madec and Imbard (1996).
}

discussed in Sect. 3.2. With the tripolar grid, we use a 7200s time step. In general, the tripolar grid has proven to be a very effective gridding of the global ocean, and we have successfully used it in various GFDL ocean models (e.g. Gerdes et al., 2005) both in MOM4.0 and the Hallberg Isopycnal Model (HIM) (Hallberg, 1997).

\subsection{Horizontal grid resolution}

Many features of the ocean circulation occur on very small spatial scales. Boundary currents such as the Gulf Stream and Kuroshio are less than $100 \mathrm{~km}$ in width, and the dynamics that determine their separation points likely involve even smaller spatial scales. Many key passages between ocean basins such as the Bering Strait, Indonesian Throughflow, and Faeroe Bank Channel involve channels that are very narrow. This is a special problem in B-grid models like MOM, which require passages to be two tracer points in width in order for flow to occur. For this reason alone, there is considerable motivation to refine grid resolution. However computational limitations preclude an indefinite refinement. Consequently, resolution in climate models is refined as best as possible, while still allowing for a reasonable model computational throughput. In order to perform multiple multicentury runs to investigate anthropogenic climate change, climate models must be able to run at speeds of 2-5 years/day on a given computational platform. At a nominal resolution of $1^{\circ}$, our current generation of models run at the upper end of this range.

Enhancements to the meridional resolution were made in the tropics, where meridionally narrow features such as the equatorial undercurrent play an important role in tropical dynamics and variability. Previous work in forecasting such phenomena (Latif et al., 1998; Schneider et al., 2003) has indicated that meridional resolution on the order of $1 / 3^{\circ}$ is required. The meridional resolution gradually transitions from $1^{\circ}$ at $30^{\circ}$ to $1 / 3^{\circ}$ at the equator. Figure 2 illustrates the grid spacing in the model.

\subsection{Vertical grid resolution}

The vertical grid spacing in OM3 was chosen with attention given to the model's ability to represent the equatorial thermocline as well as processes occuring in the sub-tropical planetary boundary layer. For this purpose, we placed 22 evenly spaced cells in the upper $220 \mathrm{~m}$, and added 28 more cells for the deeper ocean with a bottom at $5500 \mathrm{~m}$ (see Fig. 3).

The representation of solar shortwave penetration into the upper ocean in the presence of chlorophyll (see Sect. 2.8) may warrant even finer vertical resolution than that used here (Murtugudde et al., 2002). Other air-sea interaction processes may likewise call for increasingly refined upper ocean resolution. Unfortunately, the use of top grid cells thinner than roughly $10 \mathrm{~m}$ can lead to the cells vanishing when run 


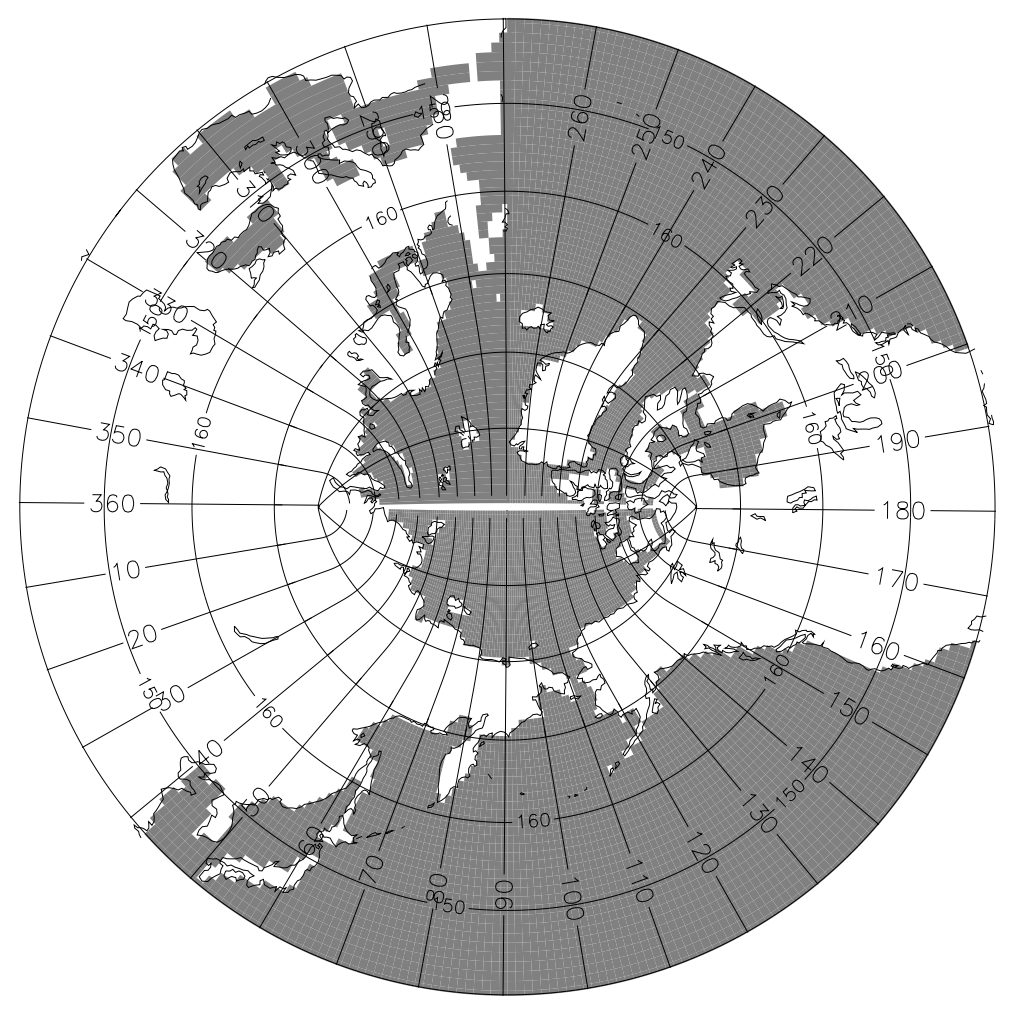

Fig. 1. Illustration of the bipolar Arctic as prescribed by Murray (1996) (see his Fig. 7) and realized in OM3. The transition from the bipolar Arctic to the spherical grid occurs at $65^{\circ} \mathrm{N}$. We denote horizontal grid cells by $(i, j)$ indices. As in the spherical coordinate region of the grid, lines of constant $i$-index move in a generalized eastward direction within the bipolar region. They start from the bipolar south pole at $i=0$, which is identified with $i=n i$, where $n i$ is the number of points along a latitude circle and $n i=360$ in OM3. The bipolar north pole is at $i=n i / 2$, which necessitates that $n i$ be an even number. Both poles are centered at a velocity point on the B-grid used in MOM4.0. Lines of constant $j$ move in a generalized northward direction. The bipolar prime-meridian is situated along the $j$-line with $j=n j$, where $n j=200$ in OM3. This line defines the bipolar fold that bisects the tracer grid. Care must be exercised when mapping fields across this fold. As noted by Griffies et al. (2004), maintaining the exact identity of fields computed redundantly along the fold is essential for model stability. Note that the cut across the bipolar fold is a limitation of the graphics package, and does not represent a land-sea boundary in the model domain.

with realistic forcing, especially with pressure loading from sea ice (see discussion in Griffies et al., 2001). Indeed, even with $10 \mathrm{~m}$ upper cells, we have found it necessary to limit the overall pressure from sea ice felt by the ocean surface to no more than that applied by $4 \mathrm{~m}$ thick ice. Ice thickness greater than $4 \mathrm{~m}$ is assumed to exert no more than $4 \mathrm{~m}$ of pressure on the sea surface.

This situation signals a fundamental limitation of free surface methods in $z$-models. In these models, only the upper grid cell feels motion of the surface height. Refined vertical cells in the presence of a realistically undulating ocean surface height requires alternative vertical coordinates (Griffies et al., 2000a). This issue is a topic of current research and development ${ }^{3}$.

\footnotetext{
${ }^{3}$ For example, the proposal by Adcroft and Campin (2004) to use the vertical coordinate of Stacey et al. (1995) for global modelling is of interest given its ability to resolve the problem of vanishing surface grid cells, while maintaining other features familiar to the $z$-models.
}

\subsection{Bottom topography}

It is common in older (those dating from before 1997) $z$ models for model grid cells at a given discrete level to have the same thickness. In these models, it is difficult to resolve weak topographic slopes without including uncommonly fine vertical and horizontal resolution. This limitation can have important impacts on the model's ability to represent topographically influenced advective and wave processes. The partial step methods of Adcroft et al. (1997) and Pacanowski and Gnanadesikan (1998) have greatly remedied this problem via the implementation of more realistic representations of the solid earth lower boundary. Here, the vertical thickness of a grid cell at a particular discrete level does not need to be the same. This added freedom allows for a smoother, and more realistic, representation of topography by adjusting the bottom grid cell thickness to more faithfully contour the topography. Figure 4 illustrates the bottom realized with the OM3 grid along the equator. Also shown is a representation using an older full step method with the same horizontal 

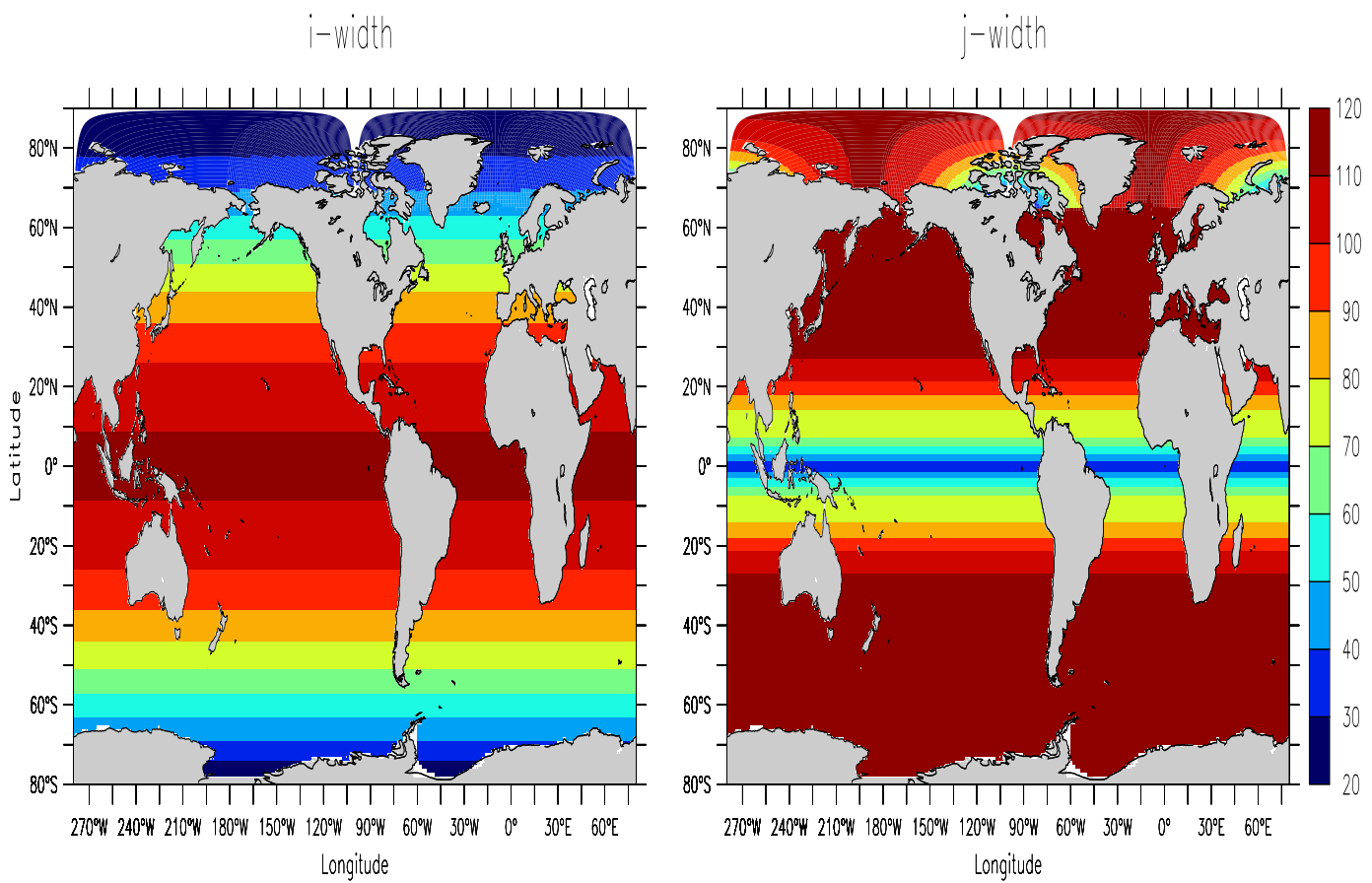

Fig. 2. Horizontal resolution of OM3 in units of kilometers. Left panel: generalized zonal (i) distance. The decrease in grid cell width moving towards the high southern latitudes is given by the cosine of the latitude. Right panel: generalized meridional (j) distance. Northward of $30^{\circ} \mathrm{S}$, the meridional spacing is refined to $1 / 3^{\circ}$ at the equator. It then coarsens again to $1^{\circ}$ at $30^{\circ} \mathrm{N}$, and stays there until reaching the bipolar region at $65^{\circ} \mathrm{N}$. There are a total of 360 zonal grid points and 200 grid points over the latitude range $78^{\circ} \mathrm{S}$ to $90^{\circ} \mathrm{N}$.

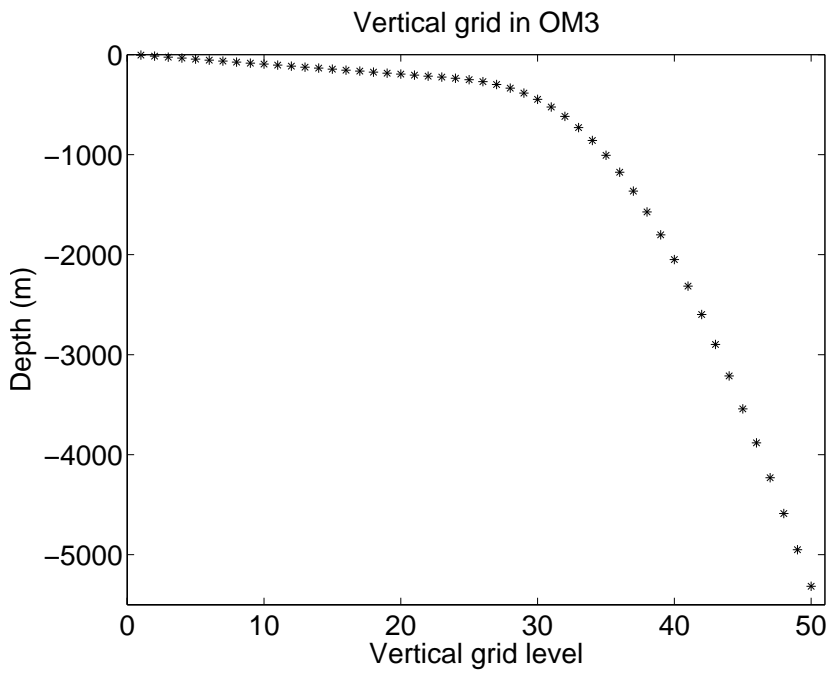

Fig. 3. Centers of the grid cells in the vertical for OM3. There are a total of 50 grid cells, with 22 evenly spaced in the upper $220 \mathrm{~m}$. The deepest ocean grid point is at $5500 \mathrm{~m}$. Deeper regions in the real ocean are relatively few and thought to be of minor consequence for climate.

and vertical resolution. The most visible differences between full step and partial step topography are in regions where the topographic slope is not large, whereas the differences are minor in steeply sloping regions.
The topography used in OM3 was initially derived from a dataset assembled at the Southampton Oceanography Centre for use in their global eddying simulations (A. Coward, personal communication). This dataset is a blend of several products. Between $72^{\circ} \mathrm{S}$ and $72^{\circ} \mathrm{N}$, version 6.2 of the satellite-derived product of Smith and Sandwell (1997) was mapped from the original Mercator projection onto a latitude-longitude grid at a resolution of 2 minutes. North of $72^{\circ} \mathrm{N}$, a version of the International Bathymetric Chart of the Oceans (Jakobssen et al., 2000) was used, while south of $72^{\circ} \mathrm{S}$ the ETOPO5 product was used (NOAA, 1988).

As mentioned in Sect. 2.2, MOM4.0 is a B-grid model in which tracer points are staggered relative to velocity points. This grid arrangement necessitates the use of no-slip sidewall conditions for realistic geometries. ${ }^{4}$ Opening channels for advective flow between basins requires the channels to be at least two tracer gridpoints wide. In the presence of complex topography not aligned with the grid, ensuring that basins which are connected in Nature are also connected within the model requires us to dig out some passages. Significant attention was paid to the North Atlantic overflows (Denmark Strait, Iceland-Scotland Overflow, Faeroe Bank Channel) based on the work of Roberts and Wood (1997) suggesting

\footnotetext{
${ }^{4}$ Topography tuning must also be combined with viscosity tuning (Sect. 3.4) due to the no-slip condition which strongly affects circulation through narrow passages.
} 


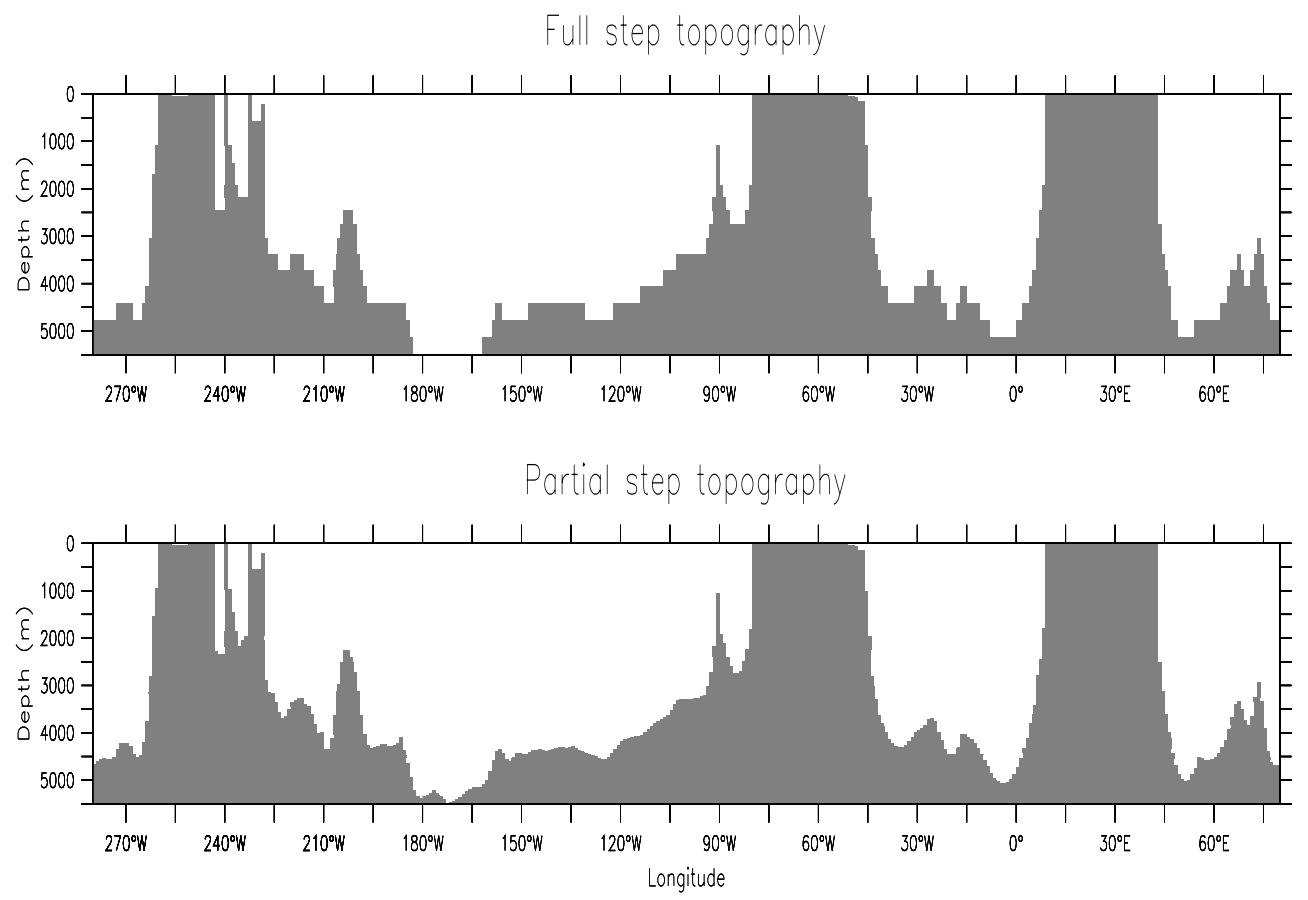

Fig. 4. Bottom topography along the equator for the tracer cells. This figure illustrates the difference between the older full step representation of the bottom topography (upper) and the partial step representation used in OM3 (lower). Note the large differences especially in regions where the topographic slope is modest and small.

that representation of the sill topography makes important differences in the ocean circulation within the Hadley Centre's climate model. Significant attention was also paid to the topography in the Caribbean Sea as well as the Indonesian Archipelago, where previous work suggests that the exact location of important islands can determine the throughflow in key passages like the Florida, Timor, and Lombok Straits (Wajsowicz, 1999). The resulting bottom depth field used in OM3 is shown in Fig. $5^{5}$.

In general, the OM3 bottom topography was arrived at via an extended multi-step process starting originally from the Southampton dataset. Unfortunately, the numerous individual steps were not completely documented, in part because of the use of early versions of the grid generation code that contained errors, and in part because of the hundreds of subjective changes. Additionally, much development work for OM3, including its topography, used a coarser resolution model (the "OM2" model used by Gerdes et al., 2005). The initial version of the OM3 topography was generated by interpolating the OM2 bathymetry to the finer OM3 grid, and was followed by the subjective modification of hundreds of individual grid depths in an effort to better represent the coastlines and the major bathymetric features (e.g. sills, ridges, straits, basin interconnections) of the World Ocean.

\footnotetext{
${ }^{5}$ This topography is available as part of a test case within the MOM4.0 distribution. Details can be found at http://www.gfdl. noaa.gov/fms.
}

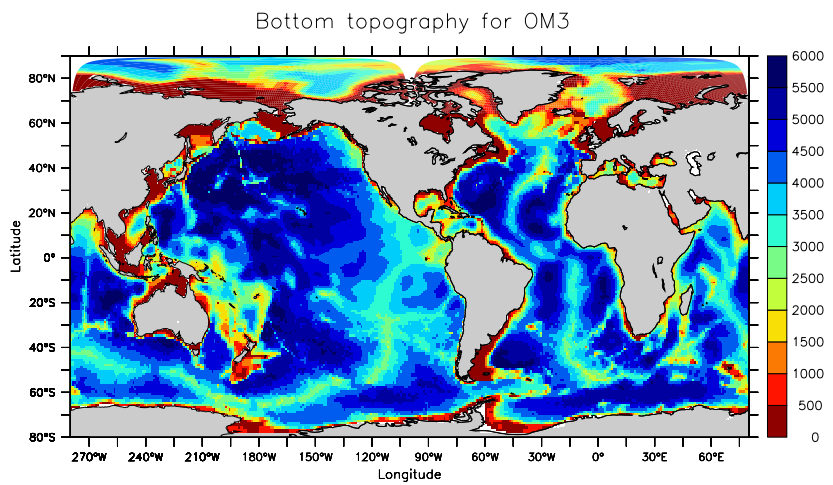

Fig. 5. The bottom depth for the tracer cells used in OM3. This topography and related html documentation is freely available as part of the MOM4.0 distribution at http://www.gfdl.noaa.gov/fms.

Before leaving the discussion of model topography, we note that in many global models from previous generations, additional numerical considerations prominently weighed in the development of a suitable topography. For example, in the commonly used rigid lid models (Bryan, 1969a), steep topography could initiate a numerical instability described by Killworth (1987), thus prompting modellers to artificially smooth ocean bathymetry. The computational cost of computing island boundary conditions (the island integrals arising in the rigid lid method) also prompted modellers to 
sink most islands in the World Ocean. Additional concerns arose from large dispersion errors contributing to unphysical tracer extrema next to rough topography, with these extrema especially prominent with second order centered advection schemes (Griffies et al., 2000b). Fortunately, these concerns are absent in the present model. Namely, the use of a free surface algorithm (Sect. 3.1) removes the rigid lid topographic instabilities and costly island integrals. The use of partial step topography (Fig. 4), and higher order dissipative tracer advection (Sect. 2.7) both reduce the presence of spurious tracer extrema.

\subsection{Bottom flows}

Partial steps do not enhance the z-model's ability to represent, or to parameterize, dense flows near the bottom which often occur in regions where the topographic slope is nontrivial. Indeed, as described by Winton et al. (1998), $z$-models used for climate rarely resolve the bottom boundary layer present in much of the World Ocean. As a result, dense water flowing from shallow marginal seas into the deeper ocean (e.g. Denmark Strait and Strait of Gibraltar), tend to entrain far more ambient fluid than observed in Nature. This spurious entrainment dilutes the dense signals as they enter the larger ocean basins, thus compromising the integrity of simulated deep water masses.

As reviewed by Beckmann (1998) and Griffies et al. (2000a), there have been various methods proposed to reduce the problems of simulating overflows in $z$-models. In OM3, we implemented the sigma diffusive element of the scheme proposed by Beckmann and Döscher (1997) and Döscher and Beckmann (2000). This scheme enhances downslope diffusion within the bottom cells when dense water lies above light water along a topographic slope.

Unfortunately, as implemented within the partial step framework, it is possible that the partial steps could become far smaller (minimum $10 \mathrm{~m}$ used here) than a typical bottom boundary layer (order 50-100 m). In such cases, the diffusive scheme is unable to move a significant amount of dense water downslope through regions with thin partial steps. A more promising method is to increase the bottom partial step minimum thickness in regions where overflows are known to be important, or to allow for the sigma diffusion to act within more than just the bottom-most grid cell. Additionally, as reported by Tang and Roberts (2005), the advective transport portion of the Beckmann and Döscher (1997) scheme provided the most significant changes in the Hadley Centre's climate model. We did not pursue these alternative approaches for OM3 due to limitations in development time. As a result, the sigma diffusion scheme has a negligible impact on the OM3's large-scale circulation, as evidenced by its very small contribution to the meridional transport of heat (not shown).

Although partial steps may be a cause for the insensitivity of the simulation to the sigma diffusion scheme, our results are consistent with those reported by Doney and Hecht
(2002), who used a similar scheme but in a model with full step bottom topography. We are uncertain whether the small impact of the overflow scheme in our climate model is related to limitations of our implementation of overflow scheme algorithm, or to problems with the surface boundary forcing. Hence, although discouraging, we believe these results warrant further focused investigation in process studies and global climate models, especially given the encouraging results from Beckmann and Döscher (1997), Döscher and Beckmann (2000), and Tang and Roberts (2005).

\subsection{Equation of state}

Ocean density is fundamental to the computation of both the pressure and physical parameterizations. Hence, an accurate density calculation is required over a wide range of temperature, salinity, and pressure. There are two methods we use to help make the calculation more accurate in CM2.

Density at a model time step $\tau$ is a function of pressure, potential temperature, and salinity at the same time step. However, in a hydrostatic model, pressure is diagnosed only once density is known. Some climate models (e.g. Bryan and Cox, 1972) resolve this causality loop by approximating the pressure used in the equation of state as $p=-\rho_{o} g z$, which is the hydrostatic pressure at a depth $z<0$ for a fluid of uniform density $\rho_{o}$. A more accurate method was suggested by Griffies et al. (2001), whereby

$\rho(\tau)=\rho[\theta(\tau), s(\tau), p(\tau-\Delta \tau)]$,

with pressure used in the equation of state lagged by a single model time step relative to potential temperature $\theta$ and salinity $s$. As recommended by Dewar et al. (1998), we include contributions from the undulating surface height and loading from the sea ice for the pressure used in the density calculation.

Previous versions of MOM used the cubic polynomial approximation of Bryan and Cox (1972) to fit the UNESCO equation of state documented in Gill (1982). This approach has limitations that are no longer acceptable for global climate modelling. For example, the polynomials are fit at discrete depth levels. The use of partial step topography makes this approach cumbersome since with partial steps, it is necessary to compute density at arbitrary depths. Additionally, the cubic approximation typically employed a narrow salinity range, which is inappropriate for many regimes of ocean climate modelling, such as wide ranges in salinity associated with rivers and sea ice. For these two reasons, a more accurate method for evaluating the equation of state is desired.

Feistel and Hagen (1995) updated the UNESCO equation of state by using more recent empirical data. In MOM4.0 we utilize a 25 term fit to their work developed by McDougall et al. (2003). The fit is valid for a very wide range of salinity, 
potential temperature, and pressure that is more than adequate for ocean climate purposes. ${ }^{6}$

\subsection{Tracer advection}

As physical climate models evolve to include chemical and biological models appropriate for the full earth system, they incorporate an increasingly wide array of tracers whose transport is greatly affected by strong spatial gradients in the presence of refined flow features. Many of the earlier compromises with tracer transport are unacceptable with these new model classes. In particular, previous versions of the GFDL ocean climate model used the second order centred tracer advection scheme. Upon recognizing that this scheme is too dispersive, later model versions incorporated the "Quicker" scheme.

Quicker is a third order upwind biased scheme based on the work of Leonard (1979), with Holland et al. (1998) and Pacanowski and Griffies (1999) discussing implementations in ocean climate models. The Quicker scheme is far less dispersive than the second order centred scheme, thus reducing the level of spurious extrema realized in the simulation. However, as with centred differences, problems can occur with unphysical tracer extrema, in particular in regions where rivers enter the ocean thus creating strong salinity gradients. Additional problems can arise with a prognostic biogeochemistry model, where even slightly negative biological concentrations can lead to strongly unstable biological feedbacks.

There are many advection schemes available which aim to remedy the above problems. Our approach for OM3 employs a scheme ported to MOM4.0 from the MIT GCM. ${ }^{7}$ The scheme is based on a third order upwind biased approach of Hundsdorfer and Trompert (1994) who employ the flux limiters of Sweby (1984). As detailed in these references, this implementation of numerical advection is nondispersive, preserves shapes in three dimensions, and precludes tracer concentrations from moving outside of their natural ranges. The scheme is only modestly more expensive

\footnotetext{
${ }^{6}$ As noted in Sect. 3 of McDougall et al. (2003), the salinity range used in the fit is 0 to $40 \mathrm{psu}$ at $0 \mathrm{db}$, but the range is reduced to 30 to $40 \mathrm{psu}$ at pressures greater than $5500 \mathrm{db}$. The minimum salinity used in the fit varies linearly with pressure from 0 psu to $30 \mathrm{psu}$ between $0 \mathrm{db}$ and $5500 \mathrm{db}$. Similarly, the maximum potential temperature used in the evaluation of the fit is $33^{\circ} \mathrm{C}$ at $0 \mathrm{db}$, varying linearly with pressure thereafter down to $12^{\circ} \mathrm{C}$ at $5500 \mathrm{db}$. The minimum potential temperature of data that is included in the evaluation of the fit corresponds to the freezing temperature at a pressure of $500 \mathrm{db}$. That is, for a given salinity, the minimum potential temperature (with a reference pressure of $0 \mathrm{db}$ ) was chosen so that if the fluid parcel was moved to a pressure of $500 \mathrm{db}$, its in situ temperature was the freezing temperature at that salinity and pressure.

${ }^{7}$ We thank A. Adcroft for assistance with this work. The online documentation of the MIT GCM at http://mitgcm.org contains useful discussions and details about this advection scheme.
}

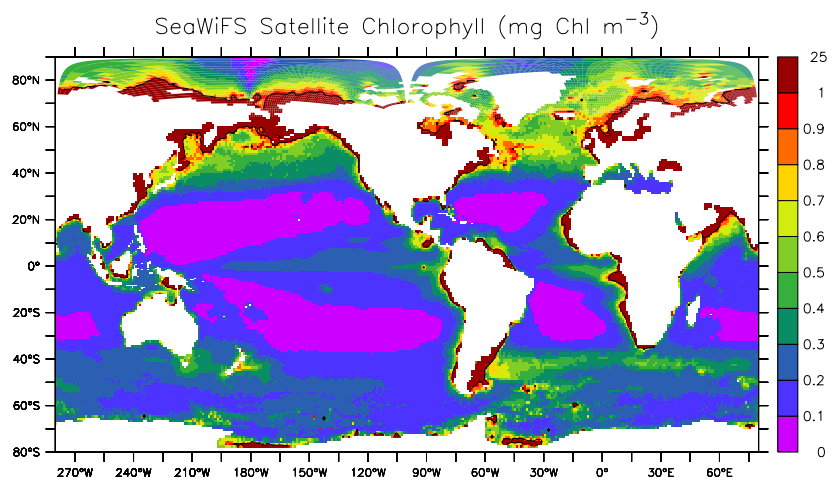

Fig. 6. Annual mean chlorophyll concentration $\left(\mathrm{mg} / \mathrm{m}^{3}\right)$ taken from the climatology developed by Sweeney et al. (2005). Note the larger values near coasts and in the polar regions are associated with high levels of biological activity in the colder and nutrient rich waters. Also, the equator is seen in both the Atlantic and Pacific as a result of increased biology in equatorial upwelling zones.

computationally than Quicker. Furthermore, we have found that it does not signficantly alter the simulation relative to Quicker in those regions where the flow is well resolved.

The question of unphysically large levels of spurious dianeutral mixing arises when considering a tracer advection scheme. Griffies et al. (2000b) document many of the issues involved. In particular, they note that so long as the admitted scales of simulated flow are well represented, levels of spurious dianeutral mixing associated with numerical advection should remain negligible.

OM3 is a mesocale eddy non-permitting model in which there are three regimes of small scale flow: (1) boundary currents, (2) tropical waves, (3) inertia-gravity waves, which are especially relevant due to the use of two hour coupling with a diurnal cycle in the climate model. The boundary current and tropical wave scales are reasonably well represented with our chosen friction and grid. The inertia-gravity waves cause density interfaces to undulate in the vertical, and the maintenance of tracer gradients in the presence of these waves can be difficult, especially in regions where the vertical grid coarsens. Griffies et al. (2000b) present a one-dimensional test problem illustrating this issue (see their Fig. 1). There, it is shown that centred second order tracer advection admits dispersive extrema that are then acted on by vertical convective adjustment. The net result is a level of spurious mixing that can be larger than that associated with third order upwind biased schemes. This result led us again to choose the Sweby scheme.

\subsection{Penetrative shortwave radiation}

The absorption of solar shortwave radiation within the upper ocean varies significantly in both space and time. High levels of chlorophyll result in almost all sunlight being absorbed within just a few meters of the ocean surface in biologically 


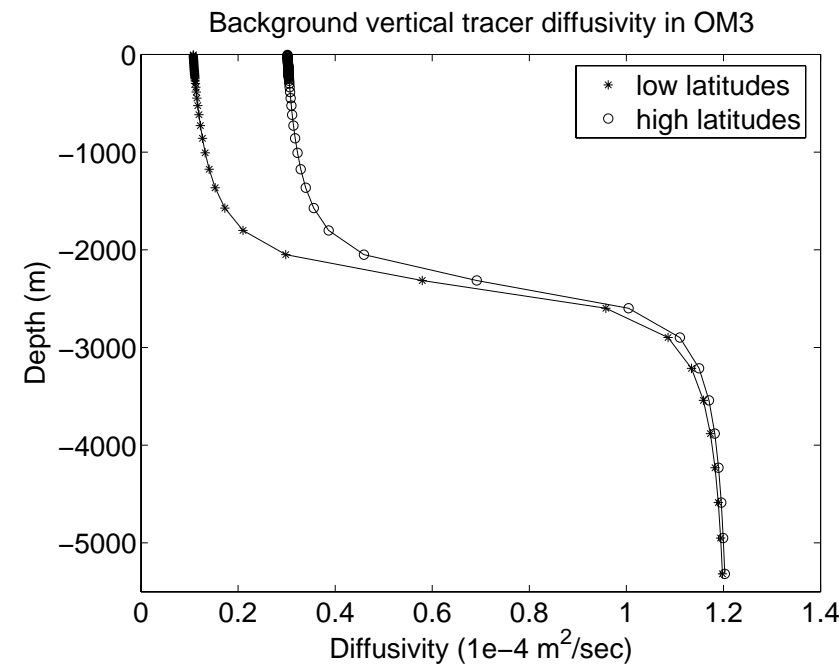

Fig. 7. Background vertical tracer diffusivity used in OM3 as suggested by Bryan and Lewis (1979). The surface values in the tropics are $0.1 \times 10^{-4} \mathrm{~m}^{2} \mathrm{~s}^{-1}$, whereas in the high latitudes they are $0.3 \times 10^{-4} \mathrm{~m}^{2} \mathrm{~s}^{-1}$.

productive waters such as near the equator, in coastal upwelling zones, and polar regions. In contrast, low chlorophyll levels in subtropical gyres allow solar radiation to penetrate with an e-folding depth (in the blue-green part of the visible spectrum) of 20-30 m.

In ocean climate models with thick upper grid cells (e.g. $50 \mathrm{~m}$ ), the geographic variation of shortwave penetration is unimportant since all shortwave radiation is generally absorbed within this single box. In OM3, however, the top box is $10 \mathrm{~m}$ with a resting ocean free surface. Up to $20 \%$ of incoming solar radiation can penetrate below this level in many regions of the ocean. Without allowing shortwave radiation to penetrate, radiative heating would overly heat the top cell, causing its temperature to grow well above observed. One way to address this problem is to allow shortwave penetration with a given e-folding depth that is constant in space and time. However, for long term global climate simulations, we believe it is important to allow geographical and seasonal variations of the shortwave penetration. Shy of a prognostic biological model, we choose a climatology rather than a global constant.

Sweeney et al. (2005) compile a seasonal climatology of chlorophyll based on measurements from the NASA SeaWIFS satellite (see Fig. 6). They used this data to develop two parameterizations of visible light absorption based on the optical models of Morel and Antoine (1994) and Ohlmann (2003). The two models yield quite similar results when used in global ocean-only simulations, with very small differences in heat transport and overturning. We use the Sweeney et al. (2005) chlorophyll climatology in CM2.0 and CM2.1 along with the optical model of Morel and Antoine (1994). Although the chlorophyll climatology remains unchanged even when considering changes in radiative forcing due to anthropogenic greenhouse gas changes, we believe it is a far better means of parameterizing shortwave penetration than available with a global constant e-folding depth. Future earth system models possessing prognostic biogeochemistry will be better able to represent potential changes in chlorophyll, and hence radiative penetration, under changing climates.

\subsection{Background vertical mixing coefficients}

Vertical tracer diffusion plays a major role in determining the overall structure of the ocean circulation, as well as its impact on climate (Bryan, 1987; Park and Bryan, 2000). Direct estimates based on measurements of temperature microstructure and the diffusion of passive tracers (Ledwell et al., 1993) indicate that the diffusivity is on the order of $0.1-0.15 \times 10^{-4} \mathrm{~m}^{2} \mathrm{~s}^{-1}$ in the extra-tropical pycnocline, and Gregg et al. (2003) indicate yet smaller values near the equator. In the deep ocean, both basin-scale budget studies (Whitehead and Worthington, 1982) and direct measurements (Toole et al., 1994, 1997; Polzin et al., $1996,1997)$ indicate that diffusivities are on the order of $1-2 \times 10^{-4} \mathrm{~m}^{2} \mathrm{~s}^{-1}$.

Until recently, most ocean climate models were unable to match the low level of diapycnal diffusivity within the pycnocline suggested from the microstructure and tracer release measurements. The reason they had problems is that some models included high values of spurious diapycnal diffusion associated with the horizontal background diffusion required to stabilize earlier versions of the neutral diffusion scheme (Griffies et al., 1998), and some had large diapycnal diffusion associated with first order upwind advection (Maier-Reimer et al., 1983). Additionally, earlier GFDL models followed Bryan and Lewis (1979) and used a vertical diffusivity of $0.3 \times 10^{-4} \mathrm{~m}^{2} \mathrm{~s}^{-1}$ in the upper ocean and $1.3 \times 10^{-4} \mathrm{~m}^{2} \mathrm{~s}^{-1}$ in the deep ocean. Higher levels of vertical diffusion within the thermocline result in an increase in tropical upwelling and poleward heat transport in both hemispheres (Gnanadesikan et al., 2003) which may compensate for the relative sluggishness of boundary currents in the coarse models.

In OM3, we maintain a relatively refined vertical resolution in the upper ocean, largely to allow for a realistically small vertical diffusivity within the tropical thermocline. Modelling experience indicates a strong sensitivity of the equatorial current structure and ENSO variability to the levels of tracer diffusion, with realistic simulations requiring small values consistent with the observations (Meehl et al., 2001).

Simmons et al. (2004) illustrate the utility of including a parameterization of mixing associated with breaking internal waves arising from the conversion of barotropic to baroclinic tidal energy. Such wave breaking occurs especially above regions of rough bottom topography (Polzin et al., 1997). The results from the Simmons et al. (2004) simulations indicate that a small value through the pycnocline and larger value at 

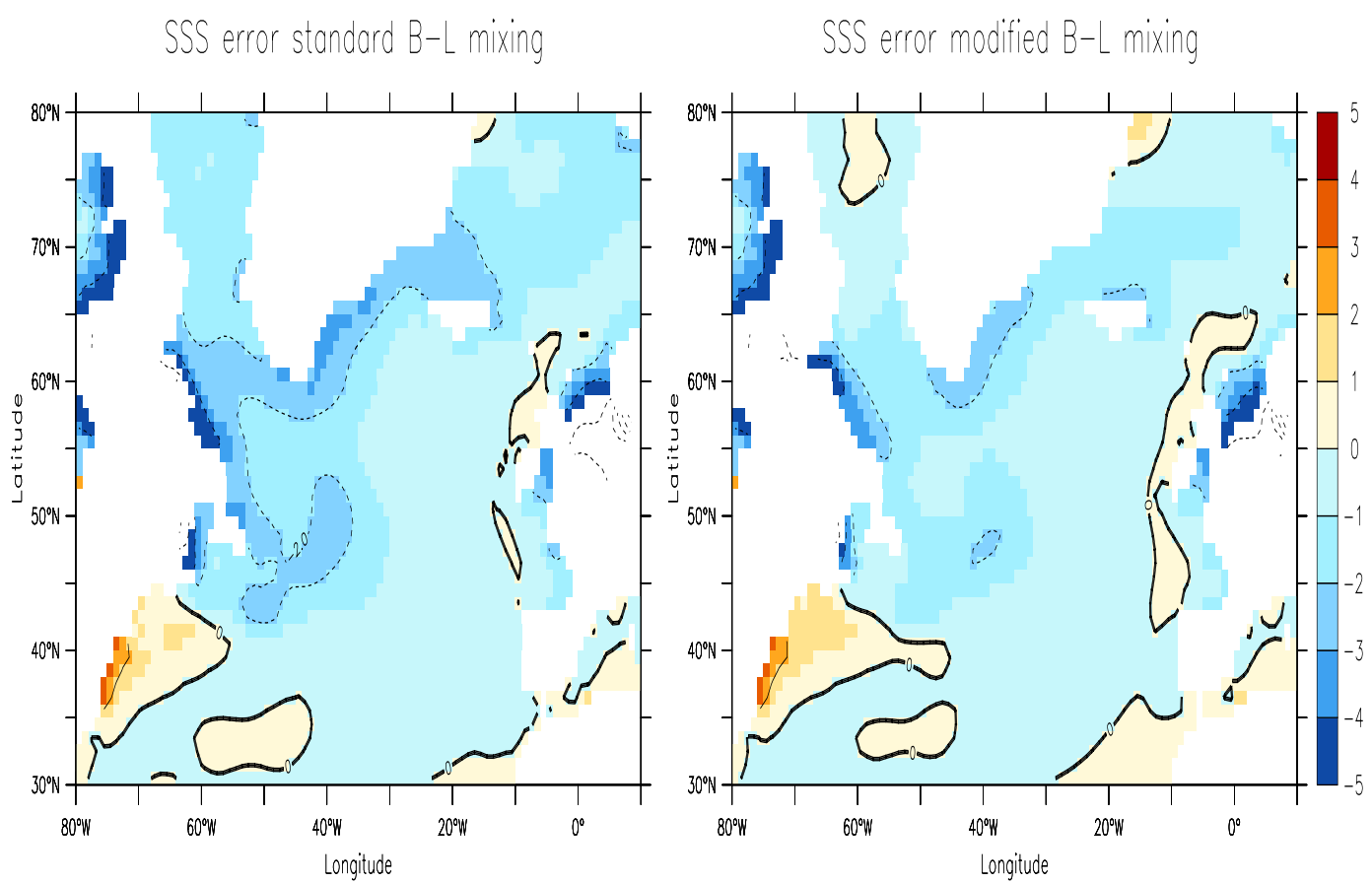

Fig. 8. Biases in the surface salinity for two runs of the CM2.0 climate model where the Bryan-Lewis background vertical diffusivity in the high latitudes is altered according to Fig. 7. The model was run for 60 years, with biases determined over years 41-60. Left panel: bias for the standard run with Bryan-Lewis tracer diffusivity the same globally. Right panel: bias using the larger upper ocean Bryan-Lewis diffusivity in the higher latitudes. Note the reduced bias in the Labrador Sea and Greenland Sea deepwater formation regions.

depth, qualitatively similar to the profile of Bryan and Lewis (1979), is far better than a vertically constant diffusivity.

While the Simmons et al. (2004) work remains the subject of much research, we decided to maintain the approach of Bryan and Lewis (1979) by prescribing a flow independent background diffusivity for OM3. To reflect the observations noted above, we modified the canonical Bryan and Lewis (1979) values to the smaller levels of $0.1 \times 10^{-4} \mathrm{~m}^{2} \mathrm{~s}^{-1}$ in the upper ocean and $1.2 \times 10^{-4} \mathrm{~m}^{2} \mathrm{~s}^{-1}$ in the deeper ocean within the tropics. In the high latitudes, we maintained the original setting of $0.3 \times 10^{-4} \mathrm{~m}^{2} \mathrm{~s}^{-1}$ in the upper ocean. Figure 7 shows the vertical profile of background vertical tracer diffusivity.

Figure 8 shows sensitivity on the North Atlantic sea surface salinity (SSS) in CM2.0 to changes in the Bryan-Lewis vertical diffusivity in the high latitudes. The larger diffusivity reduced the global RMS error in the climate model from 0.84 to 0.79 , and in the North Atlantic from 1.57 to 1.41. These are modest results, arguably not worth the cost of introducing an ad hoc latitudinal dependence to the background diffusivity.

The main goal of introducing increased tracer vertical diffusivity in the high latitudes was to address a model bias in the subpolar North Atlantic towards weak Labrador Sea deepwater formation, and a perceived fragility of simulated
Atlantic overturning ${ }^{8}$. Upon constructing CM2.1, we realized that much of this ocean bias was associated with the equatorward bias of the wind stress in the atmospheric model used in CM2.0 (see Sect. 1.3 as well as Gnanadesikan et al., 2005a; Delworth et al., 2005). Consequently, the enhanced vertical tracer diffusivity developed for CM2.0 likely was unneeded in CM2.1. Indeed, the overturning circulation is quite vigorous in CM2.1 (Delworth et al., 2005). Upon realizing this result we should have ideally returned to the vertical diffusivity tuning when constructing CM2.1 and removed the ad hoc latitudinal dependence. Unfortunately, resource and time limitations precluded this exercise. We therefore kept the same background vertical diffusivity for both CM2.1 and CM2.0.

Many modelers have traditionally taken a Prandtl number (ratio of viscosity to diffusivity) on the order 1-10. In OM3, we choose a depth independent background vertical viscosity of $10^{-4} \mathrm{~m}^{2} \mathrm{~s}^{-1}$. The level of background viscosity can also affect the equatorial currents, as discussed in Large et al. (2001). There is no theoretical or observational justification for this value of the vertical viscosity.

\footnotetext{
${ }^{8}$ Adding more diapyncal mixing generally increases the strength of the overturning (Bryan, 1987; Park and Bryan, 2000).
} 


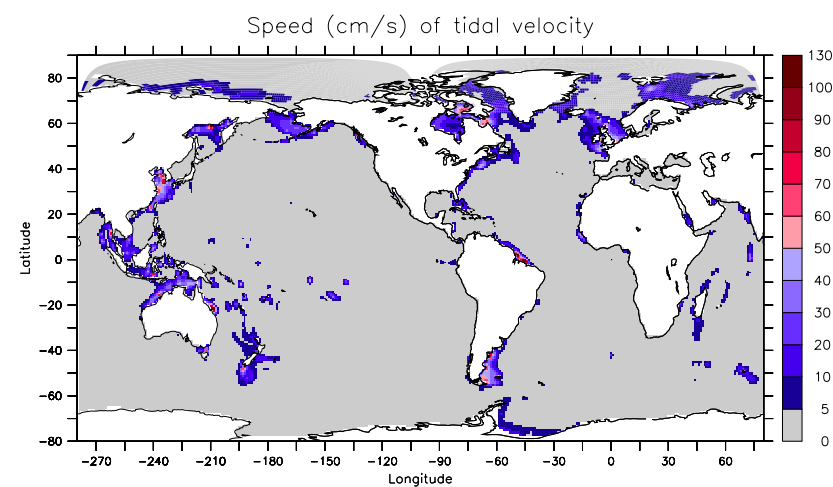

Fig. 9. Horizontal distribution of the maximum speed of the M2 tidal component from satellite data according to (Egbert et al., 1994). This speed is used to enhance the vertical shear in the computation of the Richardson number in the Large et al. (1994) boundary layer scheme in a manner described by Lee et al. (2005). Regions where the speed is high, such as near the coasts, experience enhanced mixing.

\subsection{Diapycnal mixing}

In addition to the background vertical diffusivity and viscosity discussed in Sect. 2.9, we use the parameterization of diapycnal mixing proposed by Large et al. (1994). This $k$ profile parameterization (KPP) scheme prescribes added levels of tracer and velocity mixing in regions where mixing is likely to be under-represented in this hydrostatic model, such as in the important surface ocean boundary layer. The KPP scheme has been used by many climate models during the past decade. It provides a suitable framework within which to consider various mixing processes.

Interior mixing in the ocean model is enhanced by double diffusion due to salt fingering and double diffusive convection. These processes occur in regions where the vertical temperature and salinity gradients have the same sign, and so contribute oppositely to the vertical density gradient ${ }^{9}$ (see Schmitt, 1994; Laurent and Schmitt, 1999; Toole and McDougall, 2001; Kantha and Clayson, 2000, for discussions of these processes). We follow the recommendation of Large et al. (1994) for the parameterization of diffusive convection (see their Eq. 32), yet take the alternative parameterization of

\footnotetext{
${ }^{9}$ Salt fingers can occur when warm and salty water overlies cold and fresh water (e.g. subtropical and tropical thermoclines). That is, where $\alpha \theta_{, z}>0, \beta s_{, z}>0,1<R_{\rho}<R_{\rho}^{0}$, and $R_{\rho}^{0}$ roughly equal to 2 . Here, $\alpha=-\partial_{\theta} \ln \rho$ is the thermal expansion coefficient, $\beta=\partial_{S} \ln \rho$ is the saline contraction coefficient, and $R_{\rho}=\alpha \theta_{, z} / \beta s_{, z}$ is the density ratio. Double diffusive convection occurs primarily in Arctic and adjacent regions with cold and fresh water over warm and salty water. That is, where $\alpha \theta_{, z}<0, \beta s_{, z}<0$ and $1<R_{\rho}<1$.
}

double diffusion ${ }^{10}$ given by

$$
\begin{aligned}
\kappa_{\theta} & =\kappa^{\text {other }}+0.7 \kappa_{d d} \\
\kappa_{s} & =\kappa^{\text {other }}+\kappa_{d d} \\
\kappa_{d d} & =\kappa_{d d}^{0}\left[1-\frac{R_{\rho}-1}{R_{\rho}^{0}-1}\right]^{3},
\end{aligned}
$$

where $\kappa^{\text {other }}$ is a diffusivity arising from mixing processes other than double diffusion, $\kappa_{d d}^{0}=10^{-4} \mathrm{~m}^{2} \mathrm{~s}^{-1}$, and $R_{\rho}^{0}=1.9$. This formulation is applied so long as $1<R_{\rho}<R_{\rho}^{0}$. A similar parameterization was used by Danabasoglu et al. (2005) in the recently developed Community Climate System Model, but with $R_{\rho}^{0}=2.55$. They reported a minor sensitivity of mixed layer depths to the inclusion of double diffusion (deepening of mixed layers by less than a metre). Limitations in time and resources prevented us from performing careful sensitivity tests in the GFDL model.

Another source of mixing is provided by the use of a tidal mixing parameterization for mixing along shelves. For this purpose, the Richardson number computation is modified by adding to the resolved vertical shear an unresolved shear due to tidal velocities diagnosed from a tide model according to the methods discussed in Lee et al. (2005). These tidal velocities are significant near coastal regions (see Fig. 9), in which case the Richardson numbers are small thus enhancing the vertical mixing coefficients. We found this extra mixing to be especially useful in certain river mouths to assist in the horizontal spreading of river water into the ocean basins by the horizontal currents.

\section{Novel methods and some lessons learned}

The purpose of this section is to highlight numerical and physical features of the ocean climate model that are either novel or where novel insights and experiences were garnered.

\subsection{Ocean free surface and freshwater forcing}

Variations in the ocean free surface are precluded in models using the rigid lid approximation of Bryan (1969a). This approximation was commonly made in early climate models for computational expendiency since it filters out fast barotropic undulations of the ocean free surface. However, as noted by Griffies et al. (2001), rigid lid models exhibit poor computational efficiency on parallel computers. The reason is that the elliptic problem associated with the rigid lid involves global communication across all parallel computer processors. This type of communication is costly on machines using a distributed computer processor architecture (i.e. the machines typically used for global climate modelling). Explicit free surface methods only involve less costly local processor

\footnotetext{
${ }^{10}$ Recommended to us by B. Large, personal communication, 2004.
} 
communication, which generally leads to a far more efficient algorithm.

There are physical consequences that must be considered when making the rigid lid approximation. First, the rigid lid distorts the dispersion relation for planetary waves, especially those waves with spatial scales on the order of the barotropic Rossby radius (thousands of kilometers). Second, as commonly implemented in ocean climate models, the rigid lid precludes the transport of water across ocean boundaries. The reason is that the volume of all grid cells is fixed in time, thus precluding transport of water across ocean boundaries. Hence, there is no barotropic advection giving rise to the Goldsborough-Stommel circulation, and freshwater dilution of tracer concentrations must be parameterized (see Huang, 1993; Griffies et al., 2001, and references therein for more thorough discussion of these issues).

The ocean's density, and hence its pressure and circulation, are strongly affected by the transport of water across the ocean boundaries via evaporation, precipitation, river runoff, and ice melt. That is, ocean boundaries are open to water fluxes, and these fluxes are critical to ocean dynamics. Additional climatologically important tracers, such as dissolved inorganic carbon, are also affected by water transport, as is the ocean's alkalinity.

Virtual salt fluxes used in fixed volume ocean models aim to parameterize the effects of boundary water transport on the density field. Such models transport salt, rather than water, across the air-sea interface. However, only a neglible amount of salt crosses Nature's air-sea interface. Additional virtual fluxes are required in constant volume models for other tracers. In general, virtual tracer flux methods can distort tracer changes, such as in the climatologically important situation discussed below where salinity is low as near river mouths.

Free surface methods, such as the one proposed by Griffies et al. (2001) and Griffies (2004) render the ocean volume time dependent. A time dependent ocean volume opens ocean boundaries so that water can be exchanged with other parts of the climate system. Such water transport across boundaries manifests as changes in ocean surface height (see Eq. A17). When formulated in this way, virtual tracer fluxes are inappropriate. Free surface methods also remove the distortion of barotropic planetary waves since they allow for time dependent undulations of the ocean's free surface.

Although many ocean climate models today employ a free surface algorithm for computing the vertically integrated transport and the sea surface height, tracer budgets in some models still assume the ocean volume is constant. We therefore feel it relevant to illustrate how the response of salinity to a freshwater perturbation differs in a climate model that uses virtual tracer fluxes from a model allowing water to cross its boundaries. This issue is of particular importance given the focus of climate science on changes in the hydrologic cycle and effects on the large scale thermohaline circulation.

For this purpose, consider an ocean comprised of a single grid cell affected only by surface freshwater fluxes. Conser- vation of salt in a Boussinesq model leads to

$\partial_{t}(h s)=0$

where $h$ is the cell's vertical thickness and $s$ is the salinity. In a model whose volume can change, the thickness of the ocean is altered by the addition of freshwater via

$\partial_{t} h=q_{w}$

where $q_{w}=P-E+R+I$ is the volume per horizontal area per time of precipitation, evaporation, river runoff, and net ice melting or freezing that crosses the ocean surface (Eq. A10 in the Appendix). In this case, salinity evolves according to

$h \partial_{t} s=-s q_{w}$.

For example, freshwater input to the ocean $\left(q_{w}>0\right)$ dilutes the salt concentration and so reduces salinity.

In a model using a fixed volume, salinity evolves according to

$h \partial_{t} s=-s_{\text {ref }} q_{w}$,

where now $h$ is time independent, and $s_{\text {ref }}$ is a constant salinity needed to ensure that total salt is conserved in the constant volume model assuming fresh water is balanced over the globe. ${ }^{11}$ The virtual salt flux is given by

$F^{\text {(virtual salt) }}=s_{\text {ref }} q_{w}$.

Models have traditionally taken $s_{\text {ref }}=35$, as this is close to the global averaged salinity in the World Ocean.

Use of a global constant reference salinity $s_{\text {ref }}$ distinguishes the salinity budget (Eq. 8) in the virtual salt flux model from the local salinity used in a model that exchanges water with its surroundings (Eq. 7). To illustrate how this factor alters the salinity response to freshwater forcing, consider a case where fresh river water is added to a relatively fresh ocean region where $s<s_{\text {ref }}$ (e.g. rivers discharging into the Arctic Ocean). Here, since the actual local salinity is fresher than the globally constant reference salinity, the dilution effect in the virtual salt flux model will be stronger than the real water flux model. Such overly strong feedbacks can introduce numerical difficulties (e.g. advection noise and/or salinity going outside the range allowable by the equation of state $^{12}$ ) due to unphysically strong vertical salinity gradients. For OM3, we have found problems with overly fresh waters to be particularly egregious in shelf areas of the Siberian Arctic. For the opposite case where evaporation occurs over salty

\footnotetext{
${ }^{11}$ Total salt is not conserved in constant volume models using the salinity Eq. (7) appropriate for real freshwater flux models. Nonetheless, attempts have been made at GFDL to run constant volume models with the salinity Eq. (7) in an aim to properly simulate the local feedbacks on salinity from freshwater. Unfortunately, such models tend to have unacceptably large drifts in salt content and so have not been used at GFDL for climate purposes.

${ }^{12}$ MOM4 execution is halted if temperature or salinity go outside of a specified range.
} 
regions with $s>s_{\text {ref }}$ (e.g. evaporation over subtropical gyres), the virtual salt flux model under-estimates the feedbacks onto salinity.

We now illustrate how the use of virtual salt fluxes alter the simulation characteristics in the climate model relative to real water fluxes. For this purpose, we ran two CM2.1-like experiments for a short period of time. In the standard CM2 experiments, water is input as a real water flux that affects the surface height by adding volume to the ocean fluid. For the purpose of comparison with a virtual salt flux run, we insert river water just into the top model grid cell ${ }^{13}$. We ran a second experiment with virtual salt fluxes where the virtual salt fluxes associated with the river water are applied over the top cell. Consistent with the previous theoretical discussion, results in Fig. 10 show that the virtual salt flux model has systematically fresher water near river mouths, with largest differences around 14 psu fresher. Away from rivers, the differences are minor, and consistent with variability. The virtual salt flux experiment became numerically unstable in October of the second year due to extremely unphysical values of the salinity, whereas the real water flux experiment remained stable.

In conclusion, virtual tracer fluxes can do a reasonable job of parameterizing the effects of freshwater on tracer concentration in regions where the globally constant reference tracer concentration is close to the local concentration. However, for realistic global climate models, local concentrations can deviate significantly from the global reference, especially near river mouths. This deviation compromises the physical realism and numerical stability of the simulation. These are the key reasons that we eliminated virtual tracer fluxes in our standard climate model simulations in favor of allowing water fluxes to cross the ocean model boundaries ${ }^{14}$.

\subsection{Time stepping the model equations}

Time stepping in OM3.0 is based on the standard MOM approach originating from the work of Bryan (1969a), and detailed for an explicit free surface by Killworth et al. (1991) and Griffies et al. (2001). An alternative was developed for OM3.1.

The main motivation for developing an alternative was to address tracer non-conservation associated with time filtering used to suppress the leap frog computational mode appearing in the standard method. The proposed time staggered method has much in common with that used by Hallberg (1997) for his isopycnal model, as well as by Marshall

\footnotetext{
${ }^{13}$ In the standard $\mathrm{CM} 2$ experiments, river water is inserted throughout the upper $40 \mathrm{~m}$ of the water column in a manner described in Sect. 3.6.

${ }^{14}$ The impact of virtual salt fluxes on forcing of the meridional overturning circulation in the North Atlantic is currently under investigation by researchers at GFDL (Ron Stouffer, personal communication).
}

et al. (1997) and Campin et al. (2004) for their hydrostatic and non-hydrostatic $z$-coordinate models.

The purpose of this section is to detail features of the time stepping schemes employed in OM3.0 and OM3.1. Further details are provided in Chapter 12 of Griffies (2004). We also refer the reader to the pedagogical treatments of time stepping given by Mesinger and Arakawa (1976), Haltiner and Williams (1980), and Durran (1999).

\subsubsection{The standard scheme used in OM3.0}

We start by describing the standard approach used in MOM for time stepping tracers and baroclinic velocity. For the thickness weighted tracer equation (see Sect. A2 in the Appendix for a discussion of this equation), this update takes the form

$$
\begin{aligned}
& \frac{(h T)^{\tau+1}-(\bar{h} \bar{T})^{\tau-1}}{2 \Delta \tau_{\text {leap }}} \\
= & -\nabla_{z} \cdot\left[(h \mathbf{u})^{\tau} T^{\tau, \tau-1}+h^{\tau} \mathbf{F}^{\tau-1}\right] \\
& -\delta_{k}\left[w^{\tau} T^{\tau, \tau-1}+F_{z}^{\tau+1}\right] .
\end{aligned}
$$

Here, $h$ is the time dependent thickness of a tracer cell and $T$ is the associated tracer concentration. Horizontal and vertical advection velocity components are written $(\mathbf{u}, w)$, and $\left(\mathbf{F}, F_{z}\right)$ are the horizontal and vertical SGS flux components. The horizontal gradient operator is written $\nabla_{z}$, and $\delta_{k}$ is the vertical finite difference operator acting across a discrete level $k$. Prognostic fields are updated in time increments of $\Delta \tau_{\text {leap }}$. The thickness of a tracer cell is updated analogously to the tracer, as required to maintain compatiblity between volume and tracer evolution (Griffies et al., 2001).

The time tendency in Eq. (10) has been aproximated with a centred in time discrete operator. Skipping the central time step $\tau$ introduces a spurious computational mode, where even and odd steps decouple. We choose time filtering to suppress the associated instability, with $\bar{h}$ and $\bar{T}$ denoting the time filtered thickness and tracer concentration. Absent time filtering, the discrete time tendency has a second order global truncation error, whereas time filtering degrades the truncation error to first order (see Sect. 2.3.5 of Durran, 1999). We comment further on time filtering in the subsequent discussion, as it is central to why we considered alternative time stepping schemes.

Global ocean models generally employ anisotropic grids, with significantly more refined vertical spacing than horizontal. When admitting realistically fast vertical mixing processes, parameterized by $F_{z}$, a time implicit method is used to overcome the stringent time step constraints of an explicit approach. Hence, $F_{z}$ is evaluated at the future time $\tau+\Delta \tau_{\text {leap }}$. In contrast, coarser grid spacing in the horizontal generally allows for an explicit implementation of the horizontal SGS fluxes. Due to the dissipative nature of SGS fluxes, stability considerations require them to be evaluated at the lagged time $\tau-\Delta \tau_{\text {leap }}$, with evaluation at the central time $\tau$ numeri- 


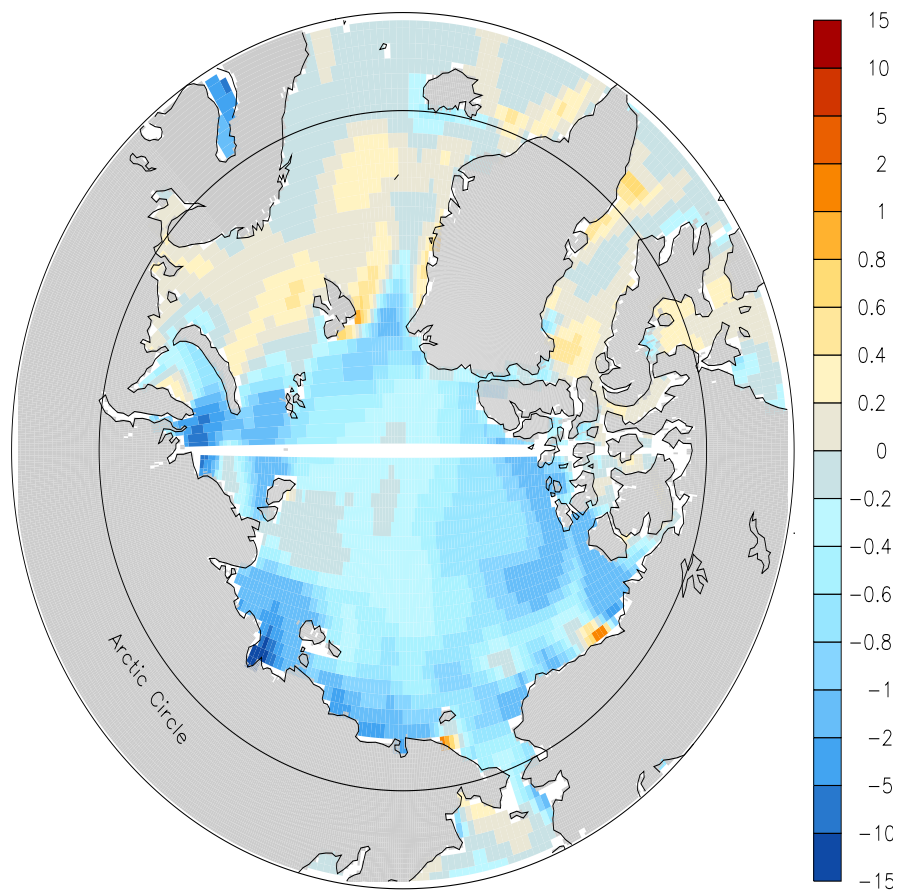

Fig. 10. Difference in Arctic surface salinity during August of the second year of integration in two CM2.1-like experiments. One experiment uses real water fluxes as in CM2.1, and the other uses virtual salt fluxes. The virtual salt flux experiment shows significantly fresher waters near river mouths, with local differences reaching 14 psu.

cally unstable. That is, the horizontal SGS fluxes are implemented with a forward time step of size $2 \Delta \tau_{\text {leap }}$.

In contrast to dissipative terms, numerical stability dictates that tracer concentration in the advection operator be evaluated at the central time $\tau$ if using central spatial differencing. As reviewed by Griffies et al. (2000a), this approach has been common in $z$-models for decades. This form of the time stepping gives rise to the commonly referred name leap frog applied to the standard time stepping used in MOM. However, it is important to note that leap frog in the tracer equation is used only for advection, and only for central spatial discretizations of advection. Dissipative terms are implemented with either a forward or an implicit time step as described above.

As discussed in Sect. 2.7, we found the dispersive errors from central differenced tracer advection to be unacceptable, due to the introduction of spurious tracer extrema and the large levels of spurious dianeutral mixing when convective adjustment acts on dispersion errors (Griffies et al., 2000b). We chose the third order upwind biased scheme discussed in Sect. 2.7 to address these issues. As reviewed in Durran (1999), upwind biasing introduces a damping or dissipative element to numerical advection. Consequently, upwind biased fluxes must be evaluated at the lagged time $\tau-\Delta \tau_{\text {leap }}$ just like the dissipative horizontal SGS fluxes. A similar situation arises when implementing the Quicker advection scheme, in which one separates a dissipative portion eval- uated at the lagged time step from a non-dissipative piece evaluated at $\tau$ (Holland et al., 1998; Pacanowski and Griffies, 1999). This is the origin of the two time labels placed on the tracer concentration for the advective flux in Eq. (10).

For the Sweby scheme used in OM3 (Sect. 2.7), the split into dissipative and non-dissipative terms is not possible. The full advective flux is thus evaluated at the lagged time step. This result may suggest increased levels of dissipation using Sweby relative to Quicker. Indeed, this is the case in regions where dissipation is welcomed, such as near river mouths where Quicker was found to introduce unacceptable tracer extrema (Sect. 2.7). In other regions of the simulation, we have seen negligible differences between the two advection schemes.

An update of the thickness weighted baroclinic velocity using the standard time stepping scheme in MOM takes the form (see Sect. A1 in the Appendix for details of the various terms)

$$
\begin{aligned}
\frac{h^{\tau+1} \mathbf{u}^{\tau+1}-\bar{h}^{\tau-1} \overline{\mathbf{u}}^{\tau-1}}{2 \Delta \tau}= & -\mathcal{M}^{\tau} \hat{\mathbf{z}} \times h^{\tau} \mathbf{u}^{\tau} \\
& +\left(w^{\tau} \mathbf{u}^{\tau}\right)_{k}-\left(w^{\tau} \mathbf{u}^{\tau}\right)_{k-1} \\
& -\nabla_{z} \cdot\left(h^{\tau} \mathbf{u}^{\tau} \mathbf{u}^{\tau}\right) \\
& -h^{\tau}(f \hat{\mathbf{z}} \times \mathbf{u})_{\text {trapezoidal }} \\
& -h^{\tau} \nabla_{z}\left(p^{\tau} / \rho_{o}\right) \\
& +h^{\tau}\left(\mathbf{F}^{\mathbf{u}}\right)^{(\tau-1, \tau+1)}
\end{aligned}
$$


As for the tracer update, time filtering is applied to the lagged values of velocity and velocity cell thickness to suppress time splitting. Central differences are used to spatially discretize velocity self-advection, thus necessitating its evaluation at the central time step. Pressure is temporally evaluated likewise. The friction operator $\left(\mathbf{F}^{\mathbf{u}}\right)^{(\tau-1, \tau+1)}$ arises from horizontal and vertical fluid deformations. Analogous to the treatment of tracer SGS fluxes, horizontal deformations are evaluated at $\tau-\Delta \tau_{\text {leap }}$ (forward time step) and vertical deformations at $\tau+\Delta \tau_{\text {leap }}$ (implicit time step).

Inertial energy is realistic in the climate model since it includes a diurnal cycle of solar insolation, and the atmosphere and sea ice fields passed to the ocean (wind stress, fresh water, turbulent and radiative fluxes) ${ }^{15}$ are updated every $2 \mathrm{~h}$. Inertial energy has important contributions to the mixing coefficients determined by the model's boundary layer scheme (Sect. 2.10).

The model's baroclinic time step is smaller than that needed to resolve inertial oscillations (e.g. Chapter 12.8.3 of Griffies, 2004). We nonetheless encountered an inertial-like instability in the climate model's Arctic sector when implementing the Coriolis force explicitly in time. This instability is presumably related to the coupling between the ocean and sea ice, although the precise mechanism remains under investigation. The climate model remained stable, however, when implementing the ocean's Coriolis force with a trapezoidal or semi-implicit method as given by Eq. (A6) in Sect. A1. Hence, this is the method employed in both OM3.0 and OM3.1. In Sect. A1, we provide more discussion of phase and amplitude errors associated with this scheme

\subsubsection{Problems related to tracer conservation}

Consider now the discrete time tracer Eq. (10) in the abbreviated form

$(h T)^{\tau+\Delta \tau_{\text {leap }}}=(\bar{h} \bar{T})^{\tau-\Delta \tau_{\text {leap }}}+2 \Delta \tau G$,

where $G$ symbolizes the advective and diffusive terms as well as boundary fluxes (we ignore source/sink terms for brevity). Thickness at the lagged time step results from a time average as described in Griffies et al. (2001), whereas time filtering of tracer concentration is taken in the form suggested by Robert (1966) and Asselin (1972) (see also Sect. 2.3.5 of Durran, 1999) ${ }^{16}$. Integrating Eq. (12) over the model domain leads to the balance of total tracer content in the model. Total tracer at time $\tau+\Delta \tau_{\text {leap }}$ is determined by the input of

\footnotetext{
${ }^{15}$ As recommended by Pacanowski (1987), wind stress applied to the ocean surface is computed using the relative velocity between the atmospheric winds and the ocean currents.

${ }^{16}$ We chose filtering for tracer over the alternative of periodically using a forward or backward time step, which was the method used by Cox (1984). The use of a periodic forward or backward time step introduces an unphysical periodicity to the simulation, and in particular was found by Marotzke (1991) to interact in unphysical ways with convective adjustment.
}

tracer through boundaries during the $2 \Delta \tau_{\text {leap }}$ time step, plus the volume integrated product of the time filtered thickness and tracer concentration, $\bar{h} \bar{T}$, at the lagged time $\tau-\Delta \tau_{\text {leap }}$. Notably, because of time filtering, the model's total tracer changes even in the case of zero boundary fluxes.

The magnitude of tracer change associated with time filtering can be negligible for many purposes, as discussed in Griffies et al. (2001). However, we found the changes unacceptable when developing ecosystem models, where precise conservation is desired. Additionally, filtering contributed to a globally averaged heat non-conservation in the climate model on the order of $\pm 0.03 \mathrm{~W} \mathrm{~m}^{-2}$. This non-conservative heat flux is a few percent of the surface insolation change expected from doubling greenhouse gas concentrations in the atmosphere. It is therefore of concern for our climate change simulations. Consequently, alternative approaches were investigated.

\subsubsection{The time staggered scheme used in OM3.1}

The alternative scheme we employ in OM3.1 discretizes the time derivative with a forward time step. That is, it does not skip any time levels. Additionally, it staggers tracer and velocity fields by one-half time step in a manner analogous to spatial staggering on Arakawa grids. We therefore refer to this method as a time staggered scheme.

Forward time stepping does not admit time splitting, and so no time filters are needed. The alternative scheme therefore ensures tracer is conserved, which is our primary motivation for moving away from the standard method involving the leap frog. There are other consequences of changing the time tendency discretization, and the purpose of this section is to expose these issues.

A time staggered update of thickness weighted tracer is given by

$$
\begin{aligned}
& \frac{(h T)^{\tau+1 / 2}-(h T)^{\tau-1 / 2}}{\Delta \tau_{\text {stag }}} \\
= & -\nabla_{z} \cdot\left[(h \mathbf{u})^{\tau} T^{\tau-1 / 2}+h^{\tau} \mathbf{F}^{\tau-1 / 2}\right] \\
& -\delta_{k}\left[w^{\tau} T^{\tau-1 / 2}+F_{z}^{\tau+1 / 2}\right] .
\end{aligned}
$$

The two Eqs. (10) and (13) become identical when (a) the time steps are related by $\Delta \tau_{\text {stag }}=2 \Delta \tau_{\text {leap }}$, (b) time filtering in the standard method is dropped, and (c) tracer advection employs an upwind biased scheme. In effect, the time staggered method stays on just one of the two leap frog branches. This is the fundamental reason that the two methods should be expected, for many purposes, to yield similar solutions.

As mentioned previously, centred spatial differencing of advection is unstable with a forward time step. Hence, for tracer advection we must employ an upwind biased scheme (Sect. 2.7). Recall that for our purposes, such advection schemes were motivated to resolve problems with other schemes. Nonetheless, this consequence of changing the 
time stepping scheme may be unacceptable for certain applications. An alternative method is to retain the ability to discretize advection with centred spatial differences, but to alter the temporal evaluation of the advection operator according to Adams-Bashforth methods (Durran, 1999), or other schemes. In particular, we chose a temporally third order accurate Adams-Bashforth method for velocity self-advection, thus maintaining the traditional centred spatial differences of this operator. The third order Adams-Bashforth method requires the advection operator at time steps $\tau, \tau-1$, and $\tau-2$, thus increasing memory requirements.

Another consequence of choosing a forward time step for the tendency is that the Coriolis force must be computed using an implicit or semi-implicit approach, such as that described in Sect. A1 (Eq. A6). In contrast, the standard approach with the leap frog allows for an explicit leap frog time stepping of the Coriolis force.

A leap frog discretization of the time tendency updates the ocean state by $\Delta \tau_{\text {leap }}$ through taking a $2 \Delta \tau_{\text {leap }}$ step for the discrete time tendency. Consequently, gravity waves and dissipative operators (i.e. diffusion, friction, and upwind biased advection) are time step constrained based on $2 \Delta \tau_{\text {leap }}$. In constrast, the staggered scheme updates the ocean state by $\Delta \tau_{\text {stag }}$ and it employs $\Delta \tau_{\text {stag }}$ to compute tendencies. It is therefore time step constrained based on a $\Delta \tau_{\text {stag }}$ time step. Hence, the staggered time step $\Delta \tau_{\text {stag }}$ can generally be twice that of the leap frog $\Delta \tau_{\text {leap }}$

$\Delta \tau_{\text {stag }}=2 \Delta \tau_{\text {leap }}$.

The computational cost of OM3.1 with the staggered scheme is therefore one-half that of OM3.0 using the standard scheme.

\subsubsection{Sensitivity to the time stepping scheme}

During the bulk of our development, the ocean model employed the standard time stepping scheme for tracer, baroclinic, and barotropic equations. Upon developing the staggered time stepping scheme for the tracer and baroclinic equations, we became convinced that the modified scheme has utility for our climate modelling applications. The question arose whether switching time stepping schemes would require retuning of the physical parameterizations.

Tests were run with the ocean and ice models using an annually repeating atmospheric forcing with daily synoptic variability, again repeating annually. Runs using the staggered scheme had a two hour time step for both tracer and baroclinic momentum, and a predictor-corrector scheme (e.g. Killworth et al., 1991; Griffies, 2004) for the barotropic equations with a $90 \mathrm{~s}$ time step ${ }^{17}$. The comparison was made to the standard time stepping scheme using one hour time

\footnotetext{
${ }^{17} \mathrm{We}$ found the predictor-corrector to be suitable for the barotropic equations due to our ability to increase the barotropic time step beyond that of the leap frog. Additionally, it preferentially dissipates grid scale features, which are commonly found when dis-
}

steps for the tracer and baroclinic equations, and (3600/64) s for the leap frog barotropic equations.

Analysis of these solutions after 10 years revealed that regions with relatively high frequency temporal variability, such as the equatorial wave guide, exhibit the most differences instantanously. Figure 11 illustrates the situation along the equator in the East Pacific. The standard simulation exhibits substantial time splitting, even with a nontrivial level of time filtering from a Robert-Asselin time filter. Moving just $5^{\circ} \mathrm{N}$ of the equator, however, reveals that the simulation has much less relative variability, and a correspondingly negligible amount of time splitting. Even though the simulation along the equator showed substantial time splitting, over longer periods of time, the large scale patterns and annual cycles showed negligible differences between time stepping schemes. Indeed, time averaging, even over just a day, seems sufficient to smooth over most of the instantaneous differences.

Tests were then run with the climate models CM2.0 and CM2.1. Instantaneous differences were much larger, as expected due to the nontrivial natural variability in the coupled system with a freely evolving atmospheric component. Nonetheless, differences for large scale patterns and seasonal or longer time averages were within levels expected from the model's natural variability.

\subsection{Neutral physics}

During the past few decades, tracer studies have shown that much of the ocean's large scale lateral transport processes are oriented according to local isopycnal directions, also known as neutral directions (e.g. McDougall, 1987), rather than surfaces of constant geopotential. Respecting this orientation has motivated the use of rotated diffusive parameterizations by Solomon (1971), Redi (1982), Olbers et al. (1985), and McDougall and Church (1986). Subsequent work by Gent and McWilliams (1990) and Gent et al. (1995) promoted the additional notion of eddy-induced advective processes (or equivalently, eddy induced skew diffusive processes). The numerical realization of these ideas in many present day $z$ models, including OM3, follows Griffies et al. (1998) and Griffies (1998). Generically, we refer to these processes as neutral physics. The purpose of this section is to explain how neutral physics appears in OM3.0 and OM3.1

The use of neutral physics in $z$-models can alter the simulation in nontrivial ways. First, it significantly reduces the unphysically large level of spurious cross isopycnal (i.e. dianeutral) mixing encountered in the older models using horizontal diffusion ${ }^{18}$. Reducing spurious mixing greatly

cretizing gravity waves on a B-grid (Killworth et al., 1991; Griffies et al., 2001). We present an analysis of the dissipative aspects in Sect. A4.

${ }^{18}$ Nontrivial problems remain for $z$-model simulations using resolutions admitting vigorous mesoscale eddies. In this case, eddies pump tracer variance to the grid scale. It has been found empiri- 

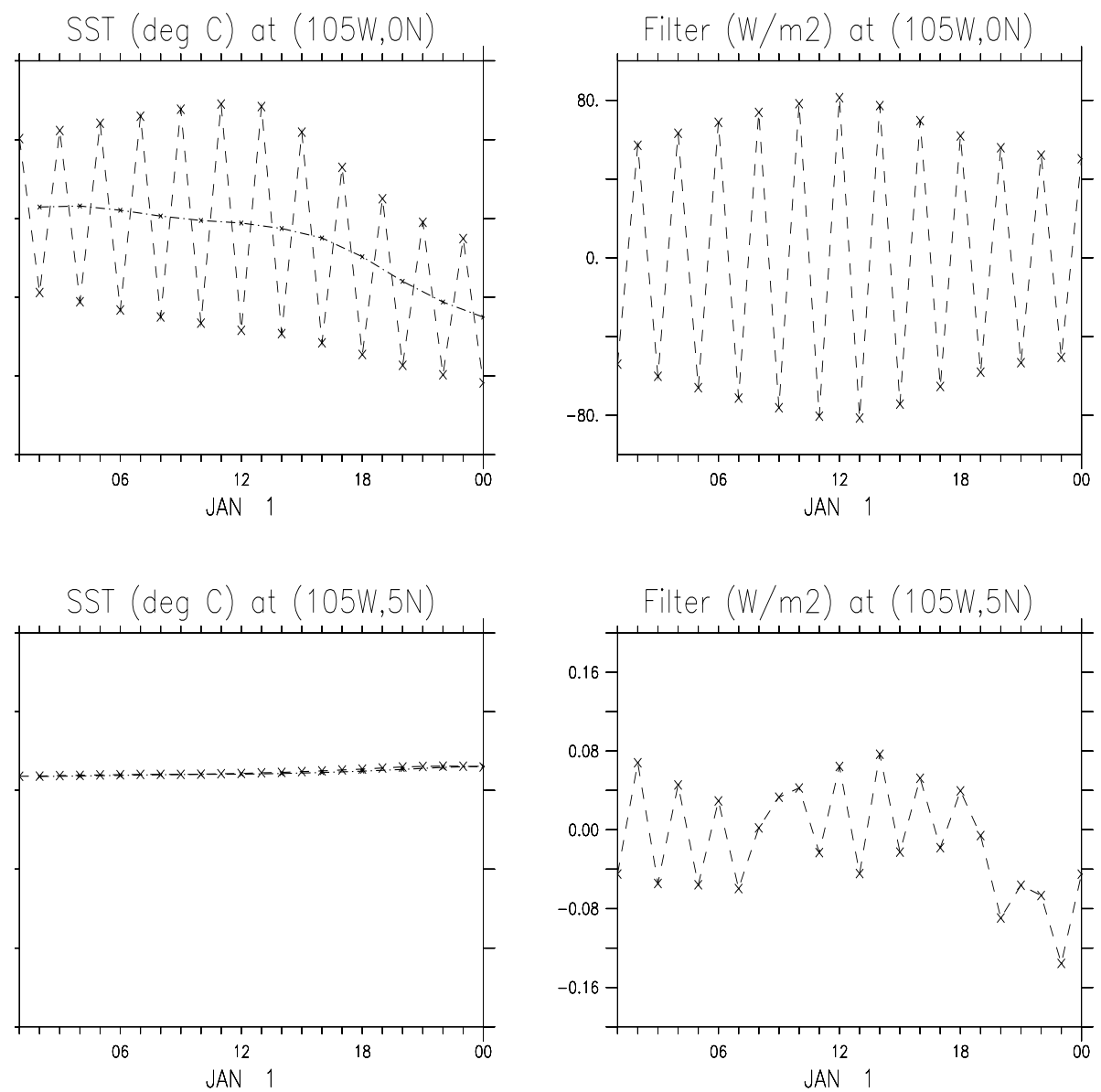

Fig. 11. Upper left panel: Instantaneous sea surface temperature over 1 January at $\left(105^{\circ} \mathrm{W}, 0^{\circ} \mathrm{N}\right)$ as realized in a simulation using the standard time stepping scheme with an hour tracer time step (noisy time series) and the staggered scheme with a two hour tracer time step (smooth time series). Upper right panel: Surface heating applied at $\left(105^{\circ} \mathrm{W}, 0^{\circ} \mathrm{N}\right)$ from the Robert-Asselin time filter used to damp the leap frog splitting. Lower left panel: Instantaneous sea surface temperature over a single day at $\left(105^{\circ} \mathrm{W}, 5^{\circ} \mathrm{N}\right)$ as realized in a simulation using the standard scheme with an hour tracer time step and the staggered scheme with a $2 \mathrm{~h}$ tracer time step. Note the width of the temperature range is set the same as at the equator. In general, the agreement of the solution off the equator, where the leap frog splitting is minimal, is far greater than on the equator. Lower right panel: Surface heating applied at $\left(105^{\circ} \mathrm{W}, 5^{\circ} \mathrm{N}\right)$ from the Robert-Asselin filter. Note the much smaller magnitude relative to the values on the equator.

improves the simulation's physical integrity, and so these schemes are ubiquitous in the ocean models participating in AR4. Use of the schemes also greatly affects the thermocline structure (Danabasoglu et al., 1994; Gnanadesikan, 1999a), heat transport (Gnanadesikan et al., 2003) and the distribution of biologically active tracers (Gnanadesikan, 1999b,c; Gnanadesikan et al., 2002).

In the following we discuss various aspects of the neutral physics as implemented in OM3.0 and OM3.1. For this

cally that this variance cannot be dissipated using traditional methods without incurring significant levels of spurious dianeutral mixing (Roberts and Marshall, 1998; Griffies et al., 2000b). It remains for $z$-modelers to empirically prove that their mesoscale eddying simulations can integrate over climatologically relevant time scales (centuries) without incurring unacceptable levels of spurious mixing. purpose it is useful to refer to Eq. (A15) which provides an expression for the tracer fluxes arising from neutral physics.

The neutral diffusive aspects of OM3.0 and OM3.1 differ. For OM3.0 we take the along isopycnal (i.e. the neutral) diffusivity $A_{I}$ to be the same depth-independent function of the flow as the skew-diffusivity (to be described later in this section). Setting the diffusivities to be the same is common practice in ocean climate models, as it is thought that the dominant physical processes parameterized by neutral diffusion and skew diffusion are associated with mesoscale eddies. Additionally, for lack of a theory stating that the diffusivities should differ, modellers have tended to take them to be the same ${ }^{19}$.

\footnotetext{
${ }^{19}$ The theoretical work of Dukowicz and Smith (1997) argue that the diffusivities should be the same.
} 
For OM3.1 we set the neutral diffusivity to the constant value of $A_{I}=600 \mathrm{~m}^{2} \mathrm{~s}^{-1}$, while keeping the skew diffusivity to be the same flow-dependent value. ${ }^{20}$ This change was motivated primarily to reduce biases in the North Pacific sea ice extent found in CM2.1, where sea ice was found to have far too large an extent in the climate simulations. In this region, isotherms and neutral directions deviate substantially. Hence, although this change in tracer mixing was not based on fundamental physical reasoning, increasing the neutral diffusivity in OM3.1 moves more heat horizontally, which reduced the overly broad sea ice extent. We illustrate these effects in Fig. 12.

For both OM3.0 and OM3.1, in regions where the neutral slope (Eq. A16) steepens, such as near the upper ocean boundary layer and within convective regions, neutral diffusion is exponentially converted to horizontal diffusion. The exponential tapering is prescribed according to the methods in Appendix B of Large et al. (1997), with our tapering started as the neutral slope becomes steeper than 1/500. Tapering also occurs in regions where the slope is less than $1 / 500$ in regions where unresolved eddies are thought to be partially cutoff because of their proximity to the ocean surface (Treguier et al., 1997; Held and Schneider, 1999). Again, the prescription given by Large et al. (1997) is followed. The region where tapering is employed is termed the neutral boundary layer in the following.

We have both physical and numerical motivation for converting neutral diffusion to horizontal diffusion within the neutral boundary layer. Physically, eddies reaching towards the upper ocean surface are kinematically constrained to transport horizontally rather than neutrally. This point was emphasized by Treguier et al. (1997), who suggested horizontal diffusion is physically proper within this region near the ocean surface.

Treatment of the skew-diffusive aspect of neutral physics in the neutral boundary layer is different from neutral diffusion. Here, the quasi-Stokes transport $-A_{g m} \mathbf{S}$ (McDougall and McIntosh, 2001; Griffies, 2004) is linearly tapered to zero starting from the boundary layer base where the magnitude of the slope $\mathbf{S}$ in either horizontal direction is just greater than 1/500. A similar method was suggested by Treguier et al. (1997) and Greatbatch and Li (2000). Because the quasi-Stokes transport is a linear function of depth within the boundary layer, the horizontal eddy velocity $\mathbf{u}^{*}=-\partial_{z}\left(A_{g m} \mathbf{S}\right)$ is vertically constant in this region (i.e. has zero vertical shear), with magnitude inversely proportional to the boundary layer depth. A generally nontrivial vertical shear in $\mathbf{u}^{*}$ is seen at the base of the boundary layer, and it is oriented in a manner to ensure the reduction of potential energy even in regions where the slope is vertical (see Sect. 15.3 of Griffies, 2004, for details) ${ }^{21}$.

\footnotetext{
${ }^{20}$ A similar approach is chosen in the Hadley Centre model (Gordon et al., 2000).

${ }^{21}$ To ensure proper orientation of the eddy velocity, it is important
}

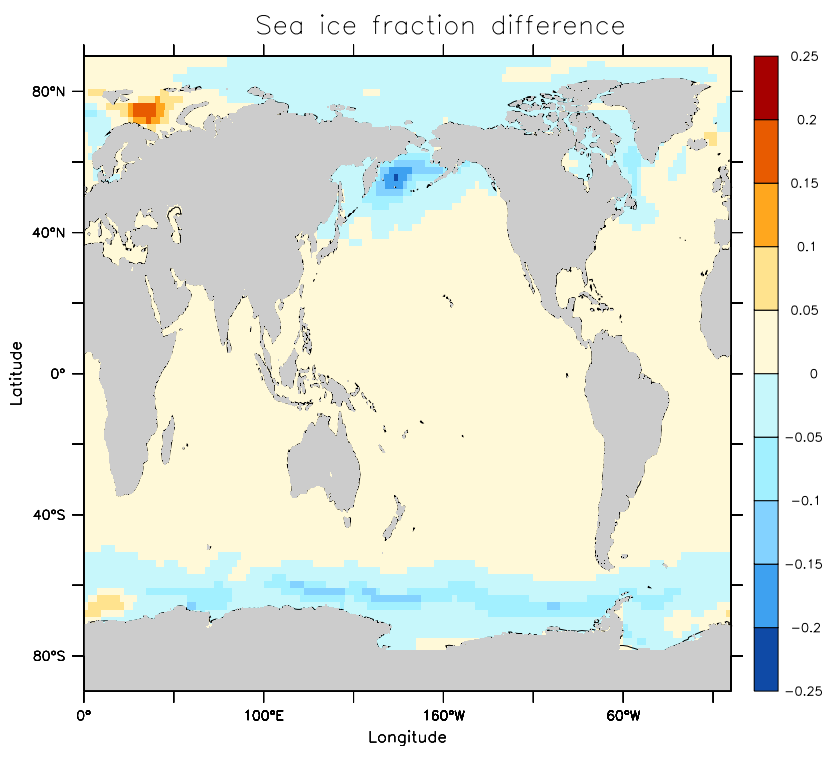

Fig. 12. Difference in sea ice fraction found in CM2.1 for the standard simulation with a constant neutral diffusivity of $A_{I}=600 \mathrm{~m}^{2} \mathrm{~s}^{-1}$ from a simulation where $A_{I}=A_{g m}$ as determined according to flow properties (as in CM2.0). The reduction in ice extent in the North Pacific found in the constant neutral diffusivity case reduced (though did not remove) biases in the coupled climate model towards too much ice in this region (see Fig. 14 of Delworth et al., 2005). Increases in ice extent in the high latitudes of the North Atlantic, however, increased model biases. Nonetheless, changes found with the constant neutral diffusivity outweighed the negatives, thus prompting the decision to use this setting in CM2.1.

Our choice of $1 / 500$ for the maximum slope parameter $S_{\max }$ is smaller than the more commonly used 1/100 (Cox, 1987), and much less than the $3 / 10$ used by Danabasoglu et al. (2005). Our reasoning for choosing this value is as follows; namely, the diffusivity times the maximum slope represents a maximum volume flux associated with the Gent and McWilliams (1990) parameterization. This product determines an upper limit on what parameterized eddies can do in countering wind-driven Ekman fluxes. Given that Ekman volume fluxes are of order $1 \mathrm{~m}^{2} \mathrm{~s}^{-1}$, we chose not to let the parameterized fluxes greatly exceed this value. The maximum skew diffusivity used in OM3 experiments is $600 \mathrm{~m}^{2} \mathrm{~s}^{-1}$, which motivated taking a maximum slope on the order of $1 / 500$.

The specific choice for the maximum slope is important especially in regions such as the Southern Ocean, where the simulation is sensitive to neutral physics details. We illustrate this sensitivity by considering the mixed layer depth. Figures 13a and 13b show mixed layer depth dif-

to maintain a non-negative squared buoyancy frequency. For this purpose, we apply the convective adjustment scheme of Rahmstorf (1993) subsequent to vertical diffusion in order to ensure that no unstable regions are acted on by the neutral physics scheme. 


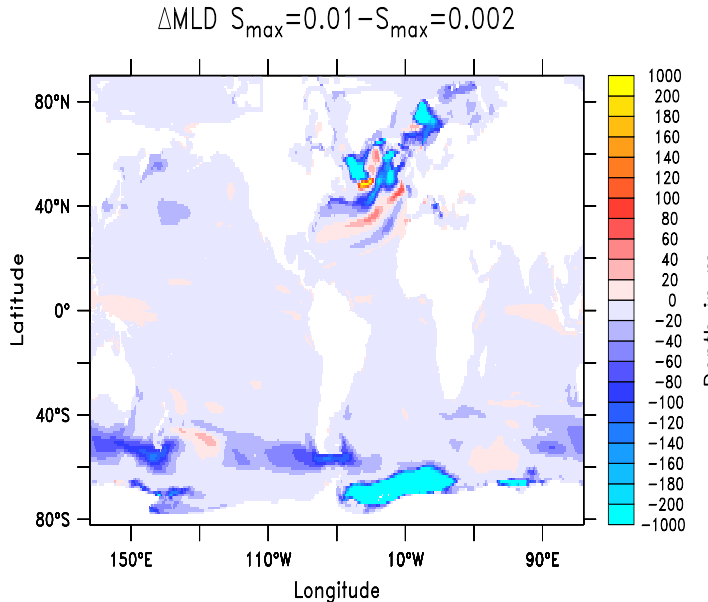

(a)

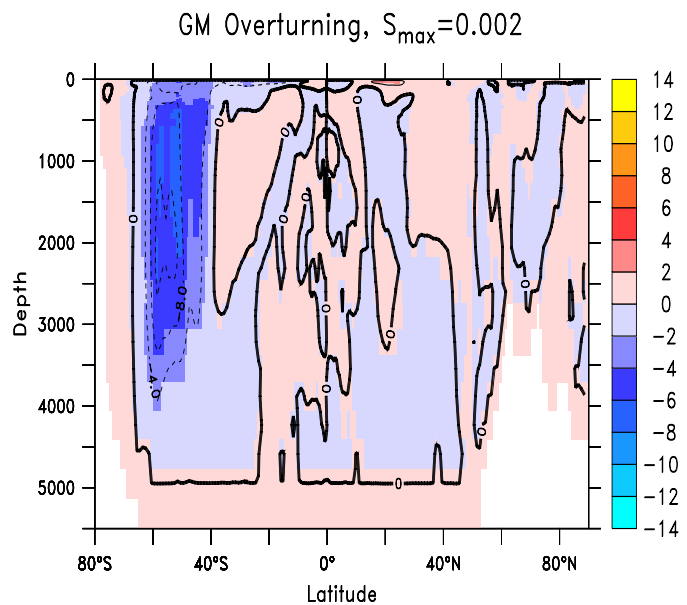

(c)
Zonally and Annually Averaged Mixed Layer Depth

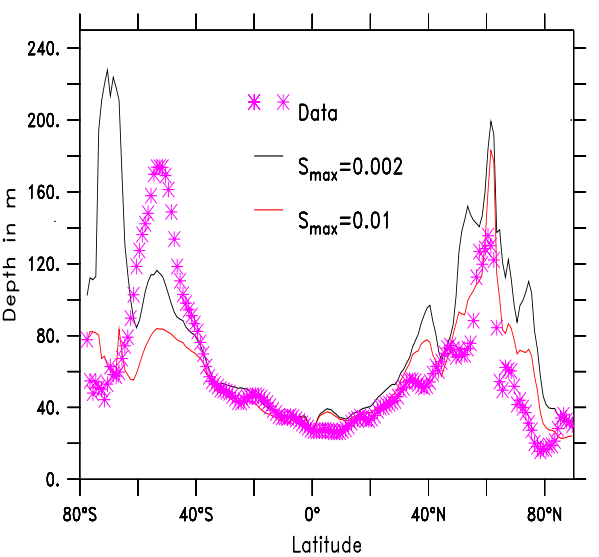

(b)

GM Overturning, $S_{\max }=0.01$

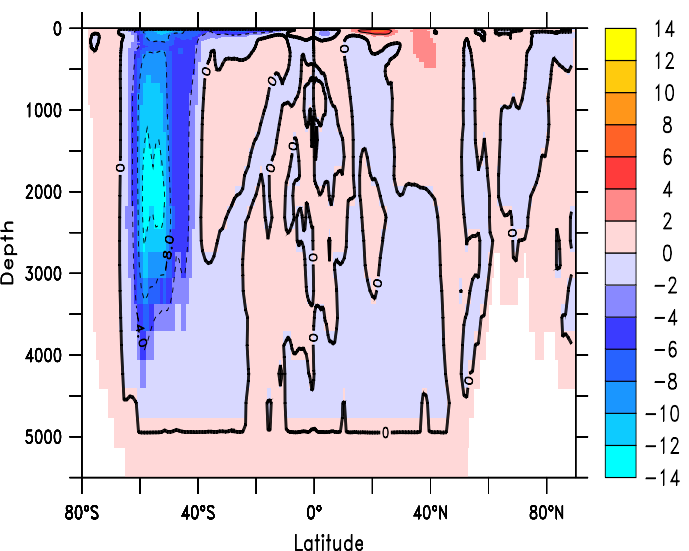

(d)

Fig. 13. Effects of changing the maximum slope above which the neutral physics schemes are exponentially tapered. Results shown here are averaged over years 40-100 of a run with CM2.1, with details reported in Gnanadesikan et al.(2005b) ${ }^{22}$. (a) Change in mixed layer depth. (b) Zonally averaged mixed layer depth compared with Conkright et al. (2002). (c) Overturning associated with the zonally integrated quasi-Stokes transport from Gent and McWilliams (1990) in units of Sv with $S_{\max }=0.002$ as in CM2.0 and CM2.1. (d) Overturning from the quasi-Stokes transport with $S_{\max }=0.01$. Note the much larger transport, especially in the Southern Ocean, with this larger value of $S_{\max }$.

ferences between a run with $S_{\max }=1 / 100$ and another with $S_{\max }=1 / 500$. The smaller $S_{\max }$ simulation generally results in decreased mixed layer depth, particularly in the Southern Hemisphere mode water formation regions and in the Labrador Sea. This behaviour illustrates how details in the neutral physics parameterization interact with the mixed layer, and thus can have a nontrivial impact on the potential vorticity structure of the mode and intermediate waters. Further discussion of this topic is given in Gnanadesikan et al. $(2005 b)^{23}$.

\footnotetext{
${ }^{23}$ Gnanadesikan, A., Griffies, S., and Samuels, B.: Effects in a climate model of slope tapering in neutral physics schemes, Ocean Modelling, submitted, 2005b.
}

The overturning streamfunction associated with the quasiStokes transport from Gent and McWilliams (1990) is also sensitive to the value of $S_{\max }$. As seen in Figs. 13c and $13 \mathrm{~d}$ this overturning is dominated by the eddy return flow which, to some extent, cancels the Deacon cell (Marshall et al., 1993; Karoly et al., 1997; Hallberg and Gnanadesikan, 2001). This circulation is much stronger when $S_{\max }=1 / 100$ than $S_{\max }=1 / 500$. Interestingly, it appears to be stronger because the slopes in the ACC are steeper.

These results illustrate that not only the value of the diffusivity, but details of how this coefficient is tapered for large neutral slopes, can produce significant changes in the largescale circulation. This fact should be remembered when considering the impact of the Gent and McWilliams (1990) 
scheme in different models. It also motivates further research into physical, rather than numerical, reasons for choosing the neutral physics tapering method (Ferrari and Plumb, 2003).

There are many methods to prescribe the diffusivity used in the neutral physics schemes. Griffies (2004) summarizes the proposals, such as Held and Larichev (1996) and Visbeck et al. (1997), that derive a depth independent diffusivity determined as a function of vertically integrated flow properties. In the construction of OM3, we tested schemes which suggest that the length scale for the diffusivity be set by the Rossby radius (Stone, 1972; Stammer, 1997; Bryan et al., 1999; Smith and Vallis, 2002). However, they generally produced far too large diffusivities within the equatorial region of the model. As this is the region of OM3 with the most refined resolution, we wish to use a tropical diffusivity that is small in order to admit flows dominated by advective, not subgrid scale, processes.

The method used in OM3 is based on setting the diffusivity proportional to the vertically averaged horizontal density gradient taken on constant depth surfaces

$A_{g m}=\alpha{\overline{\left|\nabla_{z} \rho\right|^{z}}}^{z}\left(\frac{L^{2} g}{\rho_{o} N_{o}}\right)$.

Here, $\alpha$ is a dimensionless tuning constant set to $0.07, L$ is a constant length scale set to $50 \mathrm{~km}, N_{o}$ is a constant buoyancy frequency set to $0.004 \mathrm{~s}^{-1}, g=9.8 \mathrm{~m} \mathrm{~s}^{-1}$ is the acceleration of gravity, $\rho_{o}=1035 \mathrm{~kg} \mathrm{~m}^{-3}$ is the reference density for the Boussinesq approximation, and $\left.\bar{\nabla}_{z} \rho\right|^{z}$ is the average of the horizontal density gradient taken over the depth range $100 \mathrm{~m}$ to $2000 \mathrm{~m}$ (this depth range was originally suggested by Treguier et al., 1997). A five year mean of this diffusivity from CM2.1 is shown in Fig. 14. The largest values are found in the boundary currents as well as the Antarctic Circumpolar Current. These are generally the regions where it is expected that eddy transport effects are the largest. Much smaller values are found outside these regions, as well as in the tropics. The smaller values in these regions allow for the advective dynamics resolved by the simulation to dominate the subgrid scale parameterization. Although this diffusivity has been found to be suitable for our purposes, it is unsatisfying that we cannot justify it from first principles. Thorough comparisons with alternatives remain to be conducted to clarify the utility of this approach.

As noted by Gerdes et al. (1991), truncation errors with the discrete neutral physics schemes, such as those of Griffies et al. (1998) and Griffies (1998), can cause tracer concentrations to move outside their physical bounds. This problem is similar to that arising with numerical advection schemes. To address this problem with neutral physics, Beckers et al. $(1998,2000)$ propose the use of flux limiters, analogous to those used in many advection schemes. We have not implemented neutral physics flux limiters in MOM4.0. Instead, we took a less sophisticated approach. Here, if the tracer concentration at a point moves outside a pre-defined and fixed

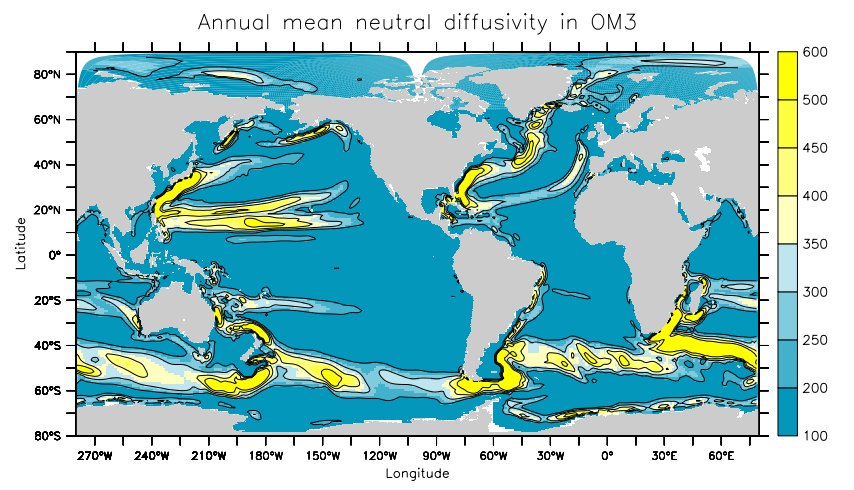

Fig. 14. Time mean diffusivity from CM2.1 over years 96-100. The minimum diffusivity is set to $100 \mathrm{~m}^{2} \mathrm{~s}^{-1}$ and maximum is $600 \mathrm{~m}^{2} \mathrm{~s}^{-1}$. This diffusivity is used just for the skew diffusivity in OM3.1. However, a similar prognostic diffusivity is used for both the neutral diffusivity and skew diffusivity in OM3.0.

global range, the tracer fluxes, instead of being those arising from neutral physics, are reduced to those from horizontal diffusion. Horizontal diffusive fluxes have been found to be needed only in special places, mostly in the high latitudes near sea ice edges and/or land/sea boundaries.

Problems can also occur with truncated neutral physics grid stencils next to the solid earth and surface boundaries. Here, the numerical realization of neutral physics parameterizations can lead to the spurious creation of extrema. To address this problem, we reduced neutral physics to horizontal diffusion at grid points adjacent to all boundaries. This approach was also recommended by Gerdes et al. (1991).

\subsection{Horizontal friction}

The ubiquitous use of horizontal friction in global ocean climate models is not motivated from fundamental physical principles. Instead, horizontal friction provides a numerical closure. In particular, it is used to maintain a modest grid Reynolds number ${ }^{24}$ and to resolve boundary currents (Griffies and Hallberg, 2000; Large et al., 2001; Smith and McWilliams, 2003; Griffies, 2004). In effect, modelers choose horizontal friction to be the smallest available given the model grid resolution and subjective notions of what constitutes a noisy simulation and/or under-resolved boundary currents. The work of Griffies et al. (2000b) also noted that simulations with under-resolved boundary currents in zmodels can be associated with unphysically large levels of spurious dianeutral tracer mixing. Such problems can cause egregious loss of water mass integrity in climate simulations. Hence, it is important to balance the desires of running a model using very low friction with the conflicting needs of ensuring that admitted flow features are well represented.

\footnotetext{
${ }^{24}$ The grid Reynolds number is $\operatorname{Re}=U \Delta / A$, where $U$ is the speed of the currents, $\Delta$ is the grid scale, and $A$ is the viscosity.
} 


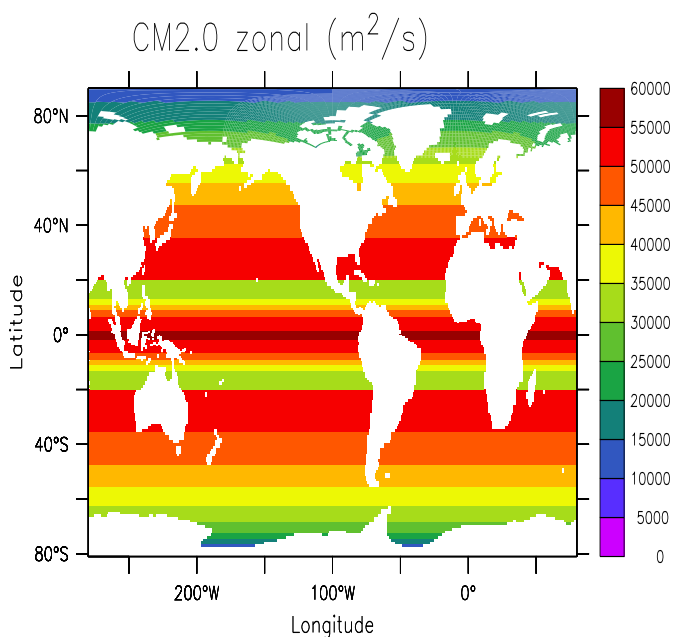

(a)

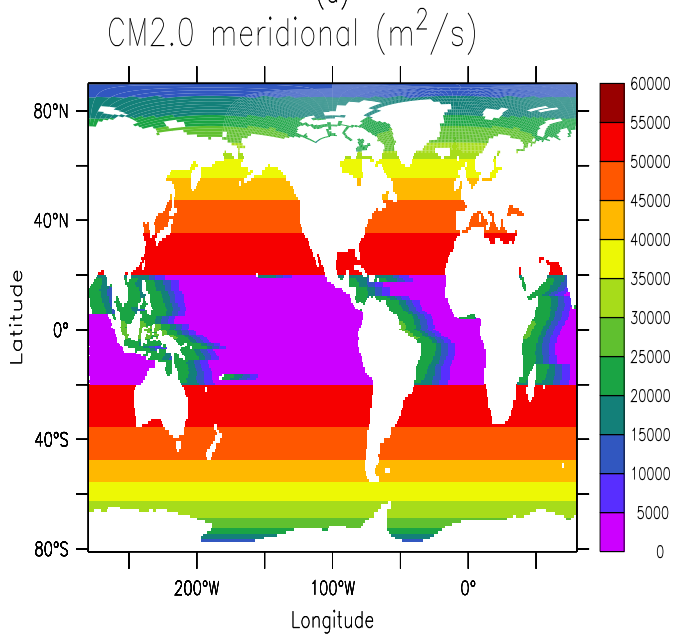

(c)

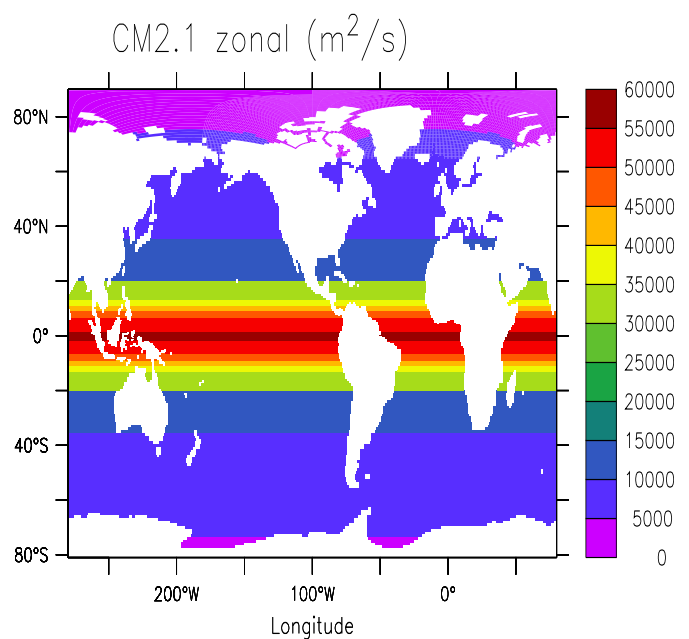

(b)

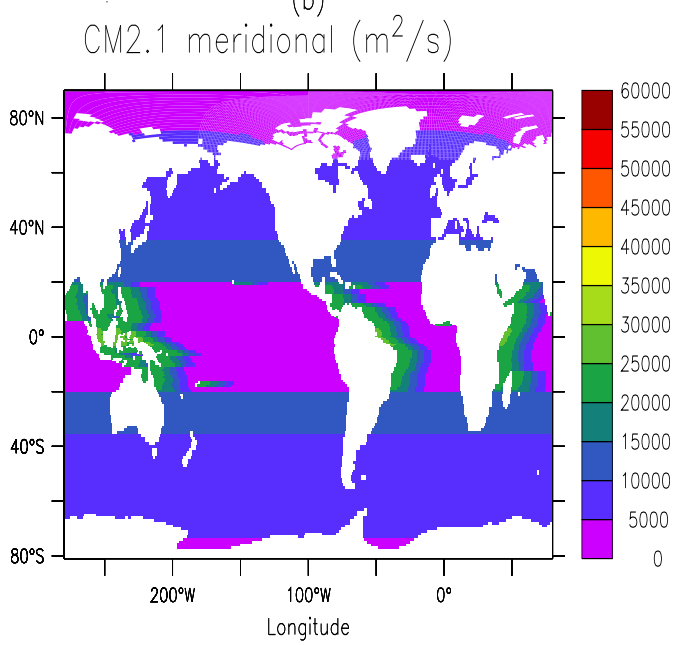

(d)

Fig. 15. Time independent zonal (upper) and meridional (lower) viscosities $\left(\mathrm{m}^{2} \mathrm{~s}^{-1}\right)$ used in OM3.0 and OM3.1 at the ocean surface. Note the values are the same in the tropics, but they are five times smaller in OM3.1 poleward of $20^{\circ}$.

Large et al. (2001) and Smith and McWilliams (2003) introduced a novel method to reduce the model's horizontal friction while satisfying the numerical needs mentioned above. Their anisotropic viscosity scheme has been employed in $\mathrm{OM} 3$ within the equatorial band from $20^{\circ} \mathrm{S}$ to $20^{\circ} \mathrm{N}$. Consistent with Large et al. (2001), the tropical current structures in OM3 are far more vigorous, and realistic, relative to the older isotropic method, and the numerical integrity of the solution is maintained (i.e. flow features are well represented, thus ensuring a negligible level of noise). Notably, the orientation of the viscosity in this region is set according to the coordinate grid lines, as suggested by Large et al. (2001). This approach ensures that larger meridional values are next to western boundaries (to resolve the Munk boundary layer) and larger zonal values are closer to the equator (to maintain a modest grid Reynolds number in the presence of strong zonal currents). Because the meridional viscosities are very small within the equatorial region, the equatorial current structures remain tight with speeds reaching to the observed $1 \mathrm{~m} \mathrm{~s}^{-1}$. Full discussion of the equatorial current structure is presented in Wittenberg et al. (2005).

Figure 15 shows the time independent zonal and meridional viscosities used in OM3.0 and OM3.1 at the ocean surface. Smaller viscosities are used at depth according to the profile suggested by Large et al. (2001). Outside of the tropics, the viscosity reverts to the traditional isotropic method, with a grid size dependent and vertically constant background viscosity added to a horizontal shear dependent Smagorinsky viscosity (Smagorinsky, 1963, 1993; Griffies and Hallberg, 2000). The Smagorinsky contribution is most noticeable in strong shear regions such as the western boundaries, but for the most part it is sub-dominant to the larger 
background viscosity shown in these figures. Additionally, to suppress a coupled ocean and sea ice instability associated with frictional CFL violations, we reduced the isotropic friction by $2 / 3$ poleward of $60^{\circ} \mathrm{N}$.

The isotropic viscosity poleward of $20^{\circ}$ is five times smaller in OM3.1 than OM3.0. To illustrate the impact in the coupled climate model, we ran the climate model CM2.1 for 100 years using an ocean component with the horizontal viscosity of OM3.0. Figure 16 shows a difference map of the 20 year mean barotropic quasi-streamfunction ${ }^{25}$. As might be expected, lowering the viscosity narrows and intensifies boundary currents. Somewhat unexpectedly, however, lowering the viscosity changes the structure of the interior gyres and overturning circulation.

The change in circulation is particularly clear in the Labrador Sea, where the Labrador gyre strengthens. Analysis of the vertical velocity shows that the lower viscosity run has much more downwelling at depth in this region, leading to a significant increase in vortex stretching in the upper water column. Associated with the increased Labrador Sea gyre is an increase in the overturning circulation (Fig. 17). Although the overturning increased signficantly, the northward heat transport only increased by a modest $0.1 \mathrm{PW}$ upon reducing the viscosity.

Increases in circulation upon lowering viscosity are also found in the weakly stratified Southern Ocean, where the ACC spins up slightly from 126.5 to $132.1 \mathrm{~Sv}$. Significant changes are found in currents through other key passages as well (Table 1), with the transports in the Florida Strait and Bering Strait significantly improved by lowering viscosity. Transports in the tropics remain relatively unchanged, as viscosity in this region remains the same.

The changes in circulation are associated with improvements in the simulated hydrography. Figure 18 compares the spatial distribution of the RMS temperature and salinity errors over the top $1500 \mathrm{~m}$ in the two runs. Clear improvements are seen in the North Atlantic, where spinup of the Labrador Sea gyre is associated with breakdown of a fresh, cold cap. Over the North Atlantic the RMS temperature error drops from $2.39^{\circ} \mathrm{C}$ to $2.17^{\circ} \mathrm{C}$, and the RMS salinity error drops from $0.73 \mathrm{psu}$ to $0.69 \mathrm{psu}$. Since the North Atlantic is the region where the RMS errors are largest, the decision was made to use the lower viscosities for CM2.1, even though doing so increases errors in other metrics, such as temperature in the North Pacific. Note that improvements in temperature error are much smaller than the changes associated with changing the winds from the different atmospheric models used in CM2.0 and CM2.1.

\footnotetext{
${ }^{25}$ As discussed in Sect. 17.2 of Griffies et al. (2004), there is no barotropic streamfunction in a free surface model since the vertically integrated transport has a nonzero divergence. However, for long time averages, the barotropic quasi-streamfunction $\psi^{(\mathrm{U})}(x, y)=-\int_{y_{o}}^{y} U\left(x, y^{\prime}\right)$ serves as a close approximation, where $U$ is the vertically integrated zonal velocity.
}

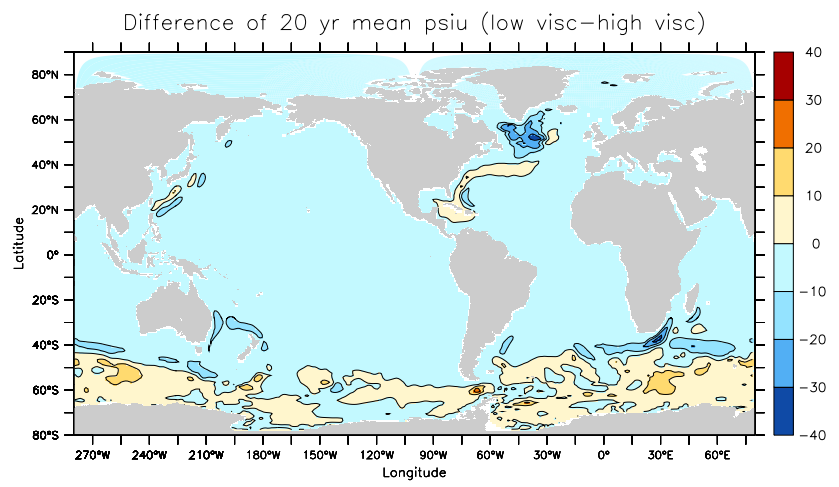

Fig. 16. Difference in the 20 year mean (years $81-100$ of the experiment) barotropic quasi-streamfunction $\psi^{(\mathrm{U})}(x, y)=-\int_{y_{o}}^{y} U\left(x, y^{\prime}\right)$ realized in CM2.1 with its horizontal viscosity, and a run with the higher viscosity used in CM2.0. The zero contour line is drawn. Note the larger transport in the Atlantic subpolar region, all boundary currents, and the Antarctic Circumpolar Current.

We close this section by noting that when attempting to lower the ocean viscosity in CM2.0, the solution worsened, particularly in the North Atlantic. Decreasing horizontal viscosity tends to move the boundary between the subpolar and subtropical gyres to the south. Since CM2.0 has wind stresses that were already shifted equatorward, decreasing the viscosity leads to an enhancement of an already strong bias. In CM2.1, a more realistic wind distribution enables us to use lower viscosities, which enabled us to improve other aspects of the circulation. This situation illustrates one of the difficulties tuning climate models, where choices made to tune one model may not apply to a different model with generally different biases and sensitivities.

\subsection{Exchange with marginal seas}

As noted in Sect. 2.2, the B-grid used in MOM4.0 requires two tracer points in order to connect ocean basins via a velocity point. This situation is problematic when marginal seas connect to the ocean through narrow passageways which are unresolved by the model grid. Such spuriously land-locked seas must be considered in the climate model in order to (a) conserve global budgets of water and tracer in the coupled climate system, and (b) allow the hydrography of the larger ocean basins to be affected by properties of the marginal seas. The Mediterranean salt tongue in the Atlantic is a canonical example where marginal sea properties strongly affect the water mass properties of a larger adjacent ocean basin.

There are two general options for handling critical unresolved passages: (a) modify the model's topography to open the passageway, thus allowing resolved transport between the marginal sea and ocean; (b) keep the marginal sea landlocked, but provide some indirect communication route. Depending on grid resolution and properties in the marginal sea, opening an unresolved passage may be quite reasonable. In 
Table 1. Vertically integrated transports and root-mean-square (RMS) errors for CM2.1 simulations using the standard low viscosity and a higher value as used in CM2.0. Statistics are based on time means computed over years 81-100. The observed Drake Passage transport is taken from Cunningham et al. (2003). Indonesian throughflow is from Gordon et al. (2003), Florida Current from Leaman et al. (1987), and Bering Strait from Roach et al. (1995). The RMS errors for potential temperature and salinity are relative to Conkright et al. (2002).

\begin{tabular}{lccc}
\hline Transport or field & observations & high viscosity & low viscosity \\
\hline Florida Straits (Sv) & $28.7-34.7$ & 17.0 & 26.9 \\
Drake Passage (Sv) & 134 & 126.5 & 132.1 \\
Bering Strait (Sv) & 0.83 & 0.57 & 0.81 \\
Indonesian Throughflow (Sv) & 10 & 14.2 & 14.1 \\
& & & \\
Global temperature (C) RMSE & 0.0 & 1.22 & 1.17 \\
Global salinity (psu) RMSE & 0.0 & 0.32 & 0.30 \\
North Atlantic temperature (C) RMSE & 0.0 & 2.39 & 2.17 \\
North Atlantic salinity (psu) RMSE & 0.0 & 0.73 & 0.69 \\
North Pacific temperature (C) RMSE & 0.0 & 1.34 & 1.38 \\
North Pacific salinity (psu) RMSE & 0.0 & 0.21 & 0.20 \\
\hline
\end{tabular}

other cases, it can result in far too much exchange between the two water bodies. The Strait of Gibraltar provides an example, where Spain is only about $12 \mathrm{~km}$ from Morrocco, thus requiring a very refined grid to explictly resolve this passage. Some coarse resolution global models, especially those based on the C-grid arrangement of model fields, successfully allow for an explicit advective flow connection between the Atlantic and Mediterranean without serious affects on the Atlantic water masses.

We chose to keep Gibraltar, and certain other passageways, closed to advective and diffusive transport in OM3, but to allow tracer exchange between the inland seas and the adjacent ocean basins through an exchange parameterization. In addition, volume exchange is available, and this is needed since moisture budgets in the climate system are generally not closed over individual basins. For example, there is a net evaporation over the Mediterranean and Red Seas. Without allowing volume to be exchanged with the Atlantic and Indian Oceans, respectively, the simulation would eventually dry up the marginal sea grid cells.

Details of the exchange parameterization, known as crossland mixing, are provided in the MOM4.0 documentation of Griffies et al. (2004). We expose here a few salient points since they have not been documented in the peerreviewed literature. For this purpose, we make reference to the schematic in Fig. 19. Consider two grid cells at the same discrete vertical point, with one inside the marginal sea and the other outside. We prescribe a conservative exchange of tracer and volume between these two cells via

$$
\begin{aligned}
\partial_{t}\left(A^{(1)} h^{(1)} T^{(1)}\right) & =\Gamma\left(h^{(2)} T^{(2)}-h^{(1)} T^{(1)}\right) \\
\partial_{t}\left(A^{(2)} h^{(2)} T^{(2)}\right) & =\Gamma\left(h^{(1)} T^{(1)}-h^{(2)} T^{(2)}\right) \\
\partial_{t}\left(A^{(1)} h^{(1)}\right) & =\Gamma\left(h^{(2)}-h^{(1)}\right) \\
\partial_{t}\left(A^{(2)} h^{(2)}\right) & =\Gamma\left(h^{(1)}-h^{(2)}\right) .
\end{aligned}
$$

In these equations, $A$ is the time independent horizontal area of a tracer grid cell, $h$ is the tracer cell thickness, $T$ is the tracer concentration, and $\Gamma$ is an exchange rate in units of area per time whose form is prescribed below. Note that the thickness $h$ has a nonzero tendency only for the top cell arising from undulations of the surface height. Furthermore, we do not allow exchange between cells adjacent to the bottom, since in this case their time independent thicknesses could be distinct due to partial step topography, in which case Eqs. (18) and (19) become inconsistent. Conservation is manifest since the total tracer and volume are constant

$$
\begin{aligned}
\partial_{t}\left(A^{(1)} h^{(1)} T^{(1)}+A^{(2)} h^{(2)} T^{(2)}\right) & =0 \\
\partial_{t}\left(A^{(1)} h^{(1)}+A^{(2)} h^{(2)}\right) & =0 .
\end{aligned}
$$

Finally, the exchange coefficient which prescribes the strength of the mixing is given by

$\Gamma=\frac{2 U}{H^{(1)}+H^{(2)}}$

where $H$ is the resting thickness of the tracer column where the exchange is prescribed, and $U$ is a prescribed volume exchange rate (units of volume per time) that is computed according to the caption to Fig. 19.

There are a total of five unresolved marginal seas in OM3 which employ the crossland mixing scheme: (1) 


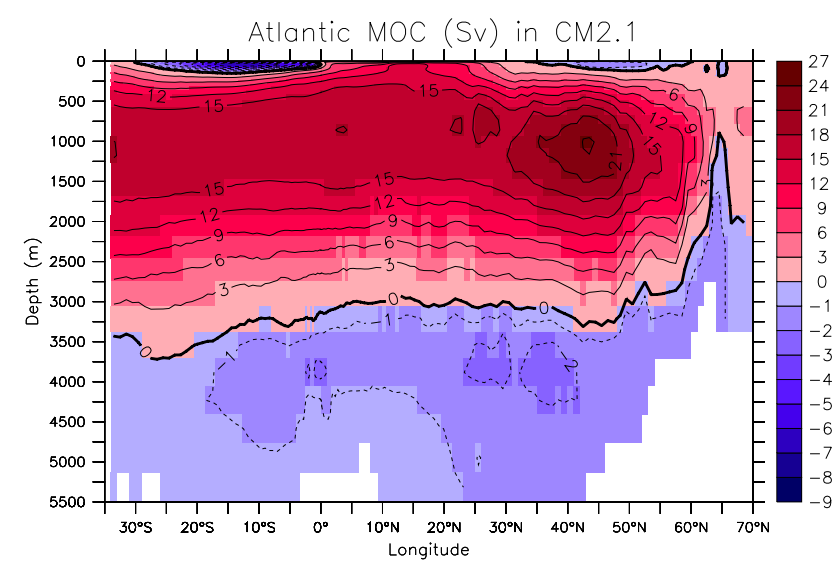

sider the situation where the prescribed exchange rates for the crossland mixing scheme are suitable for reaching an equilibrium under stable climate forcing. That is, the volume of marginal sea water is at a steady state. Now allow for changes in climate forcing to alter the fresh water budget over the catchment basin for the marginal sea (e.g. increased evaporation over the Mediterranean). In this case, it is possible for the increased evaporation to outrun the volume exchanged with the ocean basin, and so to have the top grid cells in the marginal sea dry up.

To ensure that this scenario does not occur, we added yet another pathway for volume to be exchanged. Here, we exchange volume between the two regions at a rate directly proportional to the difference in surface heights between the basins. Hence, for example, if the Mediterranean starts to dry up faster than the exchange with the Atlantic facilitated via crossland mixing, then this additional pathway available via crossland insertion extracts more water from the Atlantic and inserts it into the Mediterranean. The insertion is over a column, with vertical adjustment processes leading to a stable column at the end of the process in a manner similar to the river discharge scheme discussed in Sect. 3.6.

The motivation for including the crossland transport parameterizations is clear, given the importance of marginal seas for the World Ocean. Nonetheless, it is interesting to see what affects they have on the climate model simulation, and in particular what time scales are involved. For this purpose we configured CM2.1 in the standard way, yet removed both the crossland mixing and crossland insertion schemes. Within five years, the model reached a numerical instability because of excessive evaporation over the Red Sea causing the top model grid cell $(10 \mathrm{~m}$ thickness with a resting ocean) to dry ${ }^{27}$. In the region next to the Strait of Gibraltar on the Mediterranean side, the annual mean salinity averaged over $800-1200 \mathrm{~m}$ became quite salty (roughly $1.5 \mathrm{psu}$ saltier) within the first year relative to the World Ocean Atlas of Conkright et al. (2002). Correspondingly, over the course of the five year experiment, the region extending westward from the Iberian Penisula in the Atlantic became progressively fresher (roughly $0.5 \mathrm{psu}$ fresher) relative to Conkright et al. (2002). Both of these effects are expected in a model without transport of salty waters from the Mediterranean to the Atlantic.

\subsection{River runoff into the ocean model} $2001)^{26}$.

In addition to the crossland mixing described above, we found it essential to resolve the following difficulty associated with an ocean model whose volume can evolve. Con-

\footnotetext{
${ }^{26}$ This mode is damped in OM3.0 by the use of a Laplacian filter applied to the surface height. It is suppressed in OM3.1 by use of the predictor-corrector for the barotropic equations, which is a temporally dissipative time stepping scheme with smaller spatial scales preferentially dissipated (see Sect. A4). A biharmonic filter is also applied to the surface height in OM3.1.
}

Both the CM2.0 and CM2.1 versions of the climate model have a land component with a river routing scheme, whereby precipitation and snow melt over land are routed into the ocean at selected points. Our experience has shown that inserting the river water into the surface grid cell of the ocean

\footnotetext{
${ }^{27}$ This problem would not have occurred in a rigid lid model. Instead, salinity in the Red Sea would increase without bound absent any mixing with the fresher Indian Ocean.
} 


\section{RMS Temperature Error: $0-1500 \mathrm{~m}$}

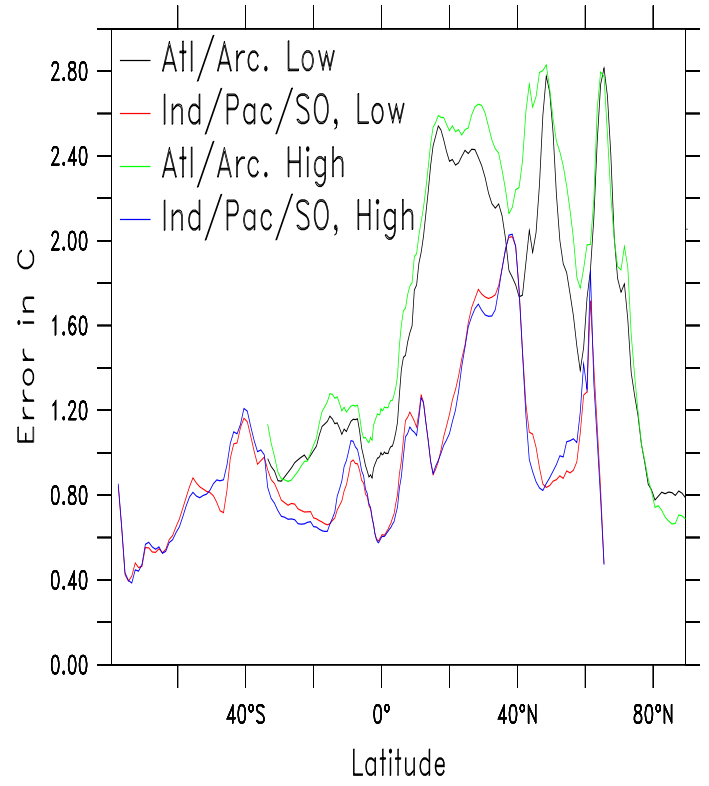

(a)
RMS Salinity Error: 0-1500m

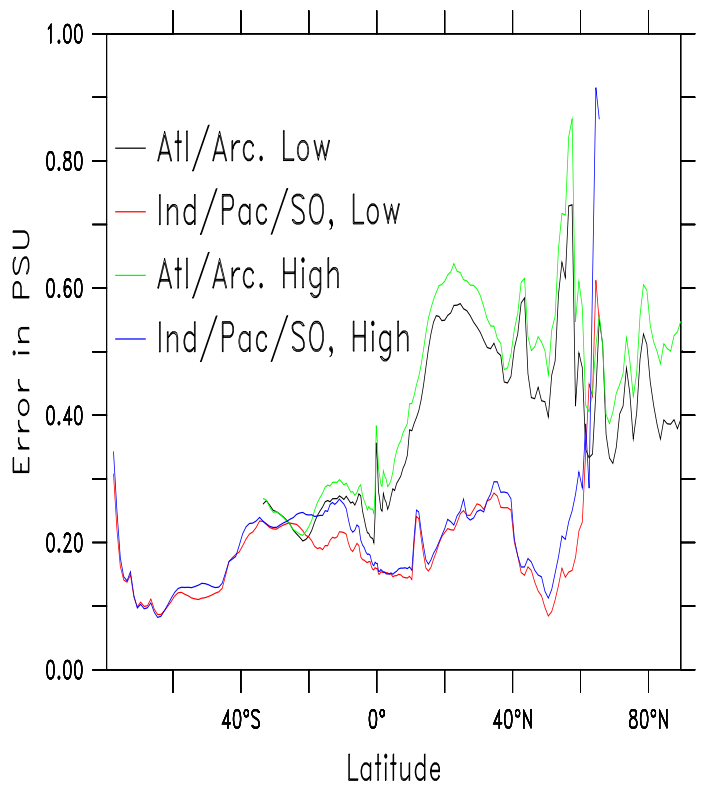

(b)

Fig. 18. Zonal mean of the root-mean-square differences over the top $1500 \mathrm{~m}$ of the low and high extratropical viscosity experiments relative to the atlas of Conkright et al. (2002). Shown here are the errors for potential temperature and salinity over various ocean basins.

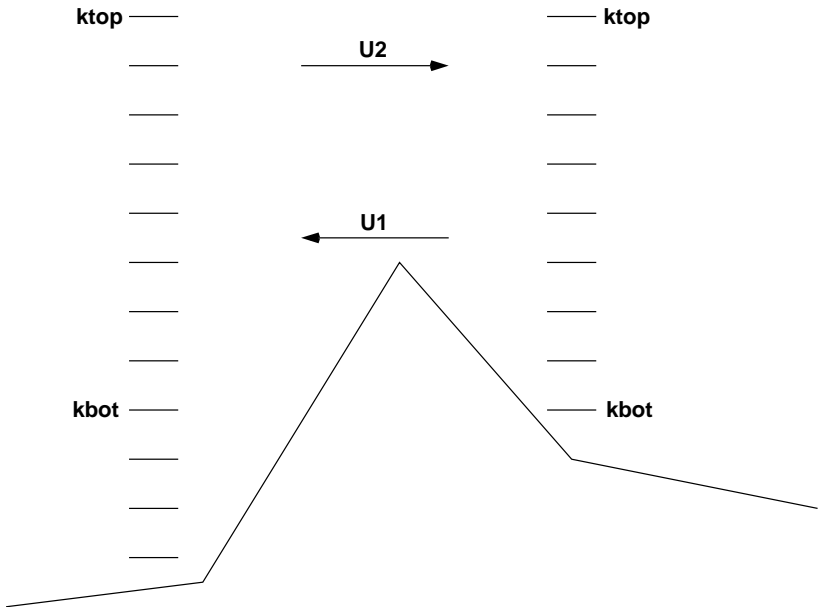

Fig. 19. Schematic of crossland mixing as implemented in MOM4.0. The model's grid mesh is assumed too coarse to explicitly represent the lateral exchange of water masses. For this schematic, we consider a subgrid scale transport $U_{1}$ moving in one direction, and $U_{2}$ in another. To represent the mixing effects on tracers by these transports, we take the exchange rate $U$ to be the average of the transports $U=\left(U_{1}+U_{2}\right) / 2$. Crossland mixing occurs between the depth levels $k=k_{\text {top }}$ and $k=k_{\text {bot }}$. If $k_{\text {top }}=1$, then crossland mixing of volume is allowed in addition to tracer transport. The transport occurs in a way to ensure conservation of both volume and tracer, as evidenced by Eqs. (20) and (21). model causes numerical problems with too much fresh water stabilizing the water column through the lighter, less dense surface waters inhibiting mixing. This problem is enhanced with refined vertical grid spacing. In reality, river plumes tend to hug the coast and are stirred by breaking waves and tidal mixing. Such mixing processes are not resolved in our model.

To reduce the salinity errors that result without sufficient coastal mixing near river mouths, some climate models spread the river discharge over a wide region near the river mouths (Danabasoglu et al., 2005). Our approach is to introduce additional mixing at the river mouth where the river routing scheme prescribed the input of river water. To partially parameterize tidal mixing, we incorporated unresolved tidal velocity shears into the KPP boundary layer scheme as discussed in Sect. 2.10. In addition, we inserted the river runoff over the upper four model grid cells (roughly $40 \mathrm{~m}$ ). In this approach, water is injected into vertical box labeled by the integer $k$, thus affecting tracer concentration within the box and causing an advective flux to the above box $k-1$. If the modified temperature and salinity profile produces an unstable density profile, convection occurs to stabilize the two boxes. Fresh water is then inserted into box $k-1$ and the process continues.

Figure 20 compares the surface salinity in two CM2.1 experiments, one run with the standard $40 \mathrm{~m}$ insertion of river runoff and the other with only $10 \mathrm{~m}$ insertion. As in Fig. 10, 


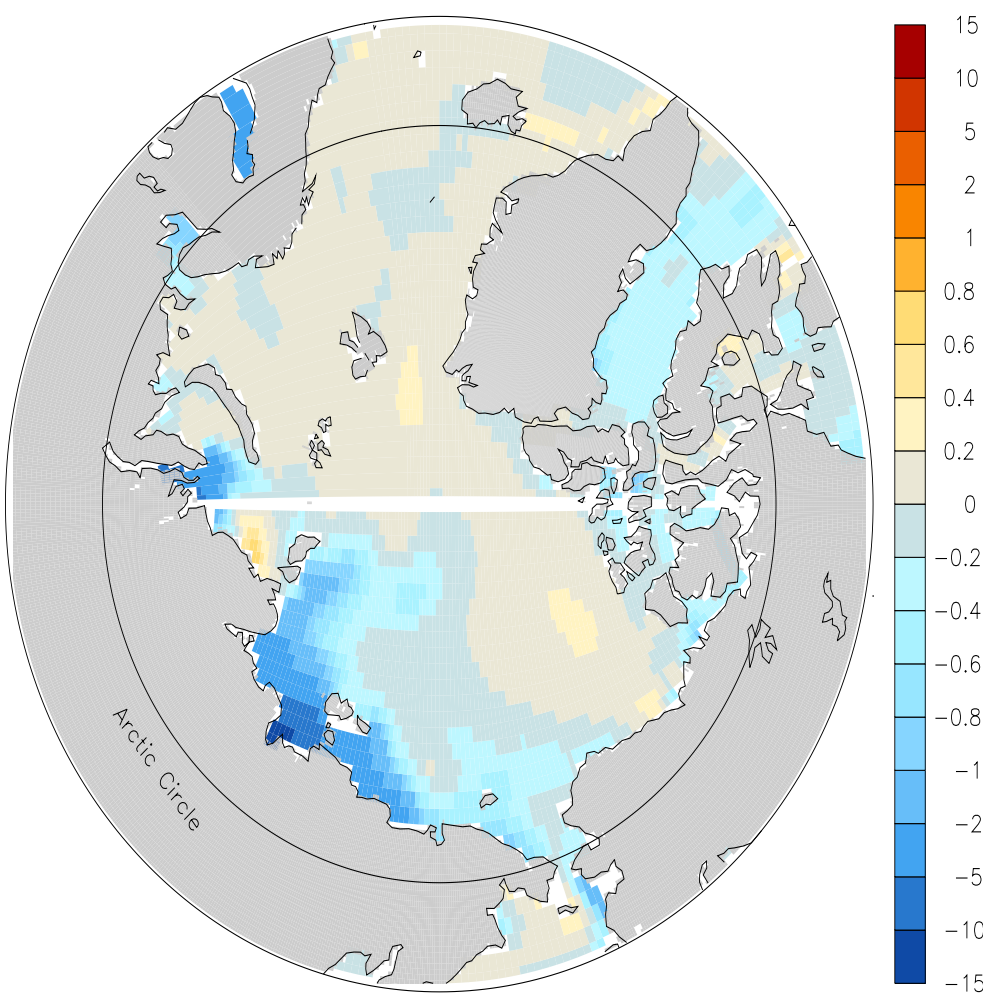

Fig. 20. Difference in surface salinity between the standard CM2.1 experiment, where river runoff is discharged over the upper $40 \mathrm{~m}$ of ocean, and test experiment with runoff discharged only over the upper $10 \mathrm{~m}$. The climate model was run for 20 years, with results from years $16-20$ shown here. Earlier pentads show analogous results, with general freshening in the Arctic using $10 \mathrm{~m}$ river insertion relative to the standard $40 \mathrm{~m}$ insertion.

we focus on the Arctic Ocean as its SSS is very sensitive to the treatment of rivers. As expected, the SSS is generally fresher in the case with only $10 \mathrm{~m}$ insertion than the standard CM2.1 experiment, with regions near river mouths noticeably fresher.

\section{Concluding remarks}

The purpose of this paper was to describe the physical and numerical algorithms used to construct the ocean component to the GFDL coupled climate model CM2. Two model versions were considered, with differences in the ocean component arising from alternative time stepping schemes and particular modifications to the lateral subgrid scale parameterizations. In general, we endeavoured to rationalize the many choices and compromises required to build a global ocean climate model. This discussion included the often omitted ad hoc steps that can be unsatisfying scientifically, but are frequently made to facilitate practical and timely advances.

There are shortcomings to what we have developed. How some of the problems affect the climate simulation are discussed in Gnanadesikan et al. (2005a). Others are associated with any ad hoc and unsatisfying approaches documented here, such as the representations of overflows, exchange with marginal seas, and the need to tune subgrid parameters without a first principles basis. Producing models that are more fully justified from first principles, either physically or numerically, is a nontrival goal that will hopefully arise with further research and development ${ }^{28}$.

Nonetheless, this document details the most realistic ocean climate model produced by GFDL. It is arguably amongst the state-of-the-art in the world today. Novel features include the following:

- Nonlinear explicit free surface with real fresh water forcing rather than virtual tracer fluxes,

- An alternative time stepping scheme that ensures tracer conservation, avoids the computational mode present with the leap frog time discretization, and allows for twice the tracer and momentum time step for our

\footnotetext{
${ }^{28}$ To facilitate the evolution of OM3 beyond that form documented here, both the ocean and sea ice components are supported by GFDL for use by the international community though the distribution of the Modular Ocean Model version 4 (MOM4.0). The code has been successfully ported to many computational platforms, and it comes with support tools and test cases of use for various modelling activities.
} 
climate model configuration, thus halving the ocean model's computational cost,

- State-of-the-art treatment of parameterized neutral physics fluxes as they interact with the surface mixed layer, and use of a novel flow dependent diffusivity to determine the strength of the fluxes.

This model has been used for century scale climate research of the coupled ocean and sea ice system, as the ocean component in the GFDL coupled climate model versions CM2.0 and CM2.1, and as the physical component of ocean biogeochemical/ecosystem models which are presently being developed for more complete earth system model purposes. Research with this model has also been conducted for seasonalinterannual forecasting and predictability, and multi-decadal global ocean analyses have been produced using a data assimilation system. Some of these applications are represented in the papers by Gnanadesikan et al. (2005a), Delworth et al. (2005), Wittenberg et al. (2005), Stouffer et al. (2005), and Russell et al. (2005) ${ }^{29}$.

The merger of diverse research and application streams represents a major advance in the collaborative use of intellectual and computational resources at GFDL, where in the past, many of these streams were represented by a broader array of models whose utility for complementary research was limited. It is likely that future advances in global ocean and climate modelling will likewise require focused efforts of numerous scientists and engineers, each providing valuable and essential contributions using a wide array of expertise, experience, and insight.

The construction of the latest GFDL coupled climate model has occupied the bulk of GFDL's intellectual and computational resources since 1999. Many other labs have recently completed similar exercises with analogous resource allocation. This time has seen tremendous improvements in computational power, software frameworks, and numerical and physical understanding of what it takes to create a coupled climate model. Many areas of modelling will certainly improve during the next round of model development. Full disclosure of the model fundamentals, facilitated by peerreviewed papers, is essential to provide a firm stepping stone towards the next model generation.

\section{Appendix A: MOM4.0 equations and methods}

The purpose of this appendix is to summarize equations and methods forming the basis for the MOM4.0 code. Some of the material here complements the focus on temporal discretization presented in Sect. 3.2. The main reference for the following material is the book by Griffies (2004) and the

\footnotetext{
${ }^{29}$ Russell, J., Stouffer, R., and Dixon, K.: Intercomparison of the Southern Ocean Circulations in the IPCC Coupled Model Control Simulations, Journal of Climate, submitted, 2005.
}

technical manual by Griffies et al. (2004), as well as other references given below.

Algorithms in MOM4.0 are based on the Boussinesq and hydrostatic approximations using surfaces of geopotential, or $\mathrm{z}$-coordinates, to discretize the vertical, and generalized orthogonal horizontal coordinates to tile the sphere. The methods for spatial and temporal discretization of the primitive equations are based on their thickness weighted form.

\section{A1 Momentum equation}

The balance of horizontal momentum per area in a Boussinesq fluid, $\rho_{o} h(u, v)=\rho_{o} h \mathbf{u}$, in a discrete grid level $k$ is written $^{30}$

$$
\begin{aligned}
{\left[\partial_{t}+(\mathcal{M}+f) \hat{\mathbf{z}} \times\right](h \mathbf{u})=} & -\nabla \cdot(h \mathbf{u} \mathbf{u})-\left(h / \rho_{o}\right) \nabla_{z} p \\
& +h \mathbf{F}+\left[w \mathbf{u}-\kappa \mathbf{u}_{, z}\right]_{k} \\
& -\left[w \mathbf{u}-\kappa \mathbf{u}_{, z}\right]_{k-1} .
\end{aligned}
$$

As commonly formulated for B-grid ocean models, this equation is written in advective form, which contrasts to the vector invariant form (see Sect. 4.4.4 of Griffies, 2004) typically used to formulate C-grid ocean models (Griffies et al., 2000a). The field $h$ is the thickness (in metres) of the model's discrete velocity cell. It is a function of space and time for the top model grid cell whose thickness changes according to undulations of the free upper surface, yet it is static for deeper cells. We now further describe terms appearing in this equation and mention the discrete numerical methods used to compute them.

- The Boussinesq reference density has the value

$$
\rho_{o}=1035 \mathrm{~kg} \mathrm{~m}^{-3} \text {. }
$$

This value is convenient since the ocean density generally varies less than $2 \%$ from it (see page 47 of Gill, 1982), whereas the more traditional (e.g. Cox, 1984) reference density of $1000 \mathrm{~kg} \mathrm{~m}^{-3}$ is less accurate.

- The advective metric angular frequency (Eq. 4.49 of Griffies, 2004)

$\mathcal{M}=v \partial_{x} \ln \mathrm{d} y-u \partial_{y} \ln \mathrm{d} x$

arises from the advection of momentum on the curved space of the sphere. It takes on the familiar form $(u / R) \tan \phi$ for spherical coordinates (Bryan, 1969a), where the horizontal grid distances are $(\mathrm{d} x, \mathrm{~d} y)=R(\cos \phi \mathrm{d} \lambda, \mathrm{d} \phi)$, with $R$ the earth's radius, $\phi$ the latitude, and $\lambda$ the longitude. However, in generalized orthogonal coordinates as used in MOM4.0, $\mathcal{M}$ is specified only when coordinate distances $\mathrm{d} x$ and $\mathrm{d} y$ are set by the choice of horizontal coordinates.

\footnotetext{
${ }^{30}$ Discrete vertical labels $k$ are exposed only where needed.
} 
- Except near the equator, the advective metric angular frequency $\mathcal{M}$ is much smaller than the Coriolis parameter

$f=2 \Omega \sin \phi$,

with $\Omega=7.292 \times 10^{-5} \mathrm{~s}^{-1}$ the earth's rotation rate. The Coriolis force $-f \hat{\mathbf{z}} \times \mathbf{u}$ is naturally discretized on the B-grid, since the horizontal velocity components $(u, v)$ are both placed at the same point.

When using a leap frog time tendency, one can take an explicit in time evaluation of the Coriolis force

$-f \hat{\mathbf{z}} \times \mathbf{u} \rightarrow-f \hat{\mathbf{z}} \times \mathbf{u}^{\tau}$,

with $\tau$ the model's baroclinic time step. However, as discussed in reference to the momentum Eq. (11), we found it necessary to employ an alternative when coupling to sea ice, in which the Coriolis force is implemented as

$-f \hat{\mathbf{z}} \times \mathbf{u} \rightarrow-f \hat{\mathbf{z}} \times\left[(1-\alpha) \mathbf{u}^{\tau-1}+\alpha \mathbf{u}^{\tau+1}\right]$.

Setting $\alpha=1$ gives an implicit treatment, and $\alpha=1 / 2$ is a semi-implicit or particular "trapezoidal" implementation. When choosing the forward time step used with the time staggered scheme in OM3.1, the $(1-\alpha) \mathbf{u}^{\tau-1}$ term is converted to $(1-\alpha) \mathbf{u}^{\tau}$. Numerical stability "necessitates" that $1 / 2 \leq \alpha \leq 1$ with the forward time scheme. We choose $\alpha=1 / 2$ in both OM3.0 and OM3.1 (see discussion after Eq. 11). For representing inertial oscillations, this choice yields zero amplitude error and favorable phase errors relative to alternatives (see Sect. 2.3.2 of Durran (1999) for details).

- The thickness weighted advection of velocity

advection $=-\nabla \cdot(h \mathbf{u} \mathbf{u})+(w \mathbf{u})_{k}-(w \mathbf{u})_{k-1}$

is discretized in space using the traditional second order centred differences, with origins in the methods of Bryan (1969a) and Cox (1984). Such facilitates a convenient transfer of energy within the discrete model in a manner analogous to the continuum (Bryan, 1969a; Semtner, 1974).

At the ocean surface, the vertical transport of horizontal momentum arises from the transport of water across the ocean free surface via

$(w \mathbf{u})_{k=0}=-q_{\mathrm{w}} \mathbf{u}_{\mathrm{w}}$,

where we choose a water velocity equal to that in the adjacent top model grid cell

$\mathbf{u}_{\mathrm{w}}=\mathbf{u}_{k=1}$.
The transport $q_{\mathrm{w}}$ measures the volume per time of water crossing the ocean free surface per unit horizontal area (Eq. 3.41 of Griffies, 2004)

$q_{\mathrm{w}} \mathrm{d} A=\hat{\mathbf{n}} \cdot \hat{\mathbf{n}}_{w}(P-E+R+I) \mathrm{d} A_{\hat{\mathbf{n}}}$,

where $\mathrm{d} A_{\hat{\mathbf{n}}}$ is the area element on the free ocean surface, $\mathrm{d} A=\mathrm{d} z \mathrm{~d} y$ is the horizontal area element on the sphere, $\hat{\mathbf{n}}$ is the outward normal at the free surface, and $\hat{\mathbf{n}}_{w}$ orients the water transport. Additionally, $P>0$ for precipitation, $E>0$ for evaporation, $R>0$ for river runoff into the ocean, and $I>0$ for sea ice melting, each of which have dimensions of a velocity, or volume per time per area.

- The horizontal friction vector $\mathbf{F}$ dissipates kinetic energy, and it arises from the divergence of horizontal frictional stresses, which are proportional to the horizontal viscosity and horizontal strains. In Sect. 3.4 we consider sensitivity of the coupled climate model to two settings for horizontal viscosity. The mathematical formulation and numerical discretization of friction are detailed in Part 5 of Griffies (2004). Notably, there is no fundamental theory for friction in ocean climate models, so it is generally tuned to yield simulations with desirable properties.

- The term $-\rho_{o} \kappa \mathbf{u}_{, z}$ in principle represents the vertical downgradient flux of horizontal momentum due to unresolved SGS processes ${ }^{31}$. It dissipates kinetic energy when there are vertical shears in the fluid. The precise value of the viscosity $\kappa$ is not well known, and so its value in our simulations is determined via tuning. In $\mathrm{CM} 2, \kappa$ is specified according to a constant background of $10^{-4} \mathrm{~m}^{2} \mathrm{~s}^{-1}$ in addition to the KPP scheme discussed in Sect. 2.10.

- As noted in Sect. 2.6, the hydrostatic pressure $p$ is diagnosed from in situ density, with density diagnosed from the equation of state using knowledge of the potential temperature, salinity, as well as the previous time step's pressure (Eq. 1). The pressure is located on the tracer grid, which is coincident with density. Hence, the horizontal pressure gradient $\nabla_{z} p$ must be averaged onto the B-grid velocity location, which lies at the tracer corners. We choose second order difference and average operations.

A spatial average in one direction of a finite difference taken in the orthogonal direction leads to computational null modes. Such modes are unphysical and so should be suppressed, as done via the use of nontrivial levels of friction (Killworth et al., 1991; Griffies et al., 2001). In contrast, the use of very small or zero levels of friction

\footnotetext{
${ }^{31}$ The comma notation for partial derivative, $\partial_{z} \mathbf{u}=\mathbf{u}_{, z}$, is a shorthand used in this appendix.
} 
readily expose these modes, which appear as a checkerboard or zig-zag pattern in the velocity between alternating grid cells.

Because of the partial bottom steps used to represent topography (Fig. 4), the bottom grid cells generally have different thicknesses. Hence, there are two terms needed to compute horizontal pressure gradients in the bottom most grid cells. The first arises from the difference in pressure between the adjacent cells, and the second arises from slopes in the bottom topography. This calculation is analogous to that needed for ocean models using topography-following vertical coordinates. Importantly, however, this calculation is needed here only for the bottom-most grid cell in a particular column. Pacanowski and Gnanadesikan (1998) further detail this importanat technical point.

\section{A2 Tracer equation}

The thickness weighted tracer equation at a discrete depth level $k$ takes the following form in continuous time and continuous horizontal space

$$
\begin{aligned}
\partial_{t}(h T)= & -\nabla \cdot[h(\mathbf{u} T+\mathbf{F})]+h \mathcal{S}^{(T)} \\
& +\left(w T+F^{z}\right)_{k}-\left(w T+F^{z}\right)_{k-1} .
\end{aligned}
$$

For material tracers such as salinity and nutrients, the dimensionless tracer concentration $T$ represents the mass of tracer per mass of seawater within a parcel of fluid. Its evolution is described by the above scalar conservation law whereby its value is determined by the convergence of thickness weighted advective fluxes

$$
\begin{aligned}
\text { advection }= & -\nabla \cdot(h \mathbf{u} T) \\
& +\left(w T+F^{z}\right)_{k}-\left(w T+F^{z}\right)_{k-1},
\end{aligned}
$$

the convergence of SGS fluxes $\left(\mathbf{F}, F^{z}\right)$, and sources $\mathcal{S}^{(T)}$. Evolution of the thermodynamical tracer potential temperature $\theta$ is also described by this equation (see Chapter 5 of Griffies, 2004, for fundamentals of the tracer equation).

Discretization of advective fluxes are discussed in Sect. 2.7. Sections 2.9 and 2.10 describe parameterizations that specify the diapycnal tracer flux implemented as downgradient vertical diffusion

$F^{z}=-\kappa T_{, z}$

where $\kappa$ is a diapycnal tracer diffusivity. Finally, Sect. 3.3 outlines our approach for including SGS neutral physics processes in the simulations. We implement neutral physics according to the methods described by Griffies et al. (1998) and Griffies (1998), where the tracer flux arising from SGS neutral physics is given by

$F^{m}=-J^{m n} T_{, n}$ where the summation convention is followed. The second order SGS tracer transport tensor $J^{m n}$ is given by

$$
\mathbf{J}=\left(\begin{array}{ccc}
A_{I} & 0 & \left(A_{I}-A_{g m}\right) S_{x} \\
0 & A_{I} & \left(A_{I}-A_{g m}\right) S_{y} \\
\left(A_{I}+A_{g m}\right) S_{x}\left(A_{I}+A_{g m}\right) S_{y} & S^{2} A_{I}
\end{array}\right),
$$

where $A_{I}$ is the neutral diffusivity and $A_{g m}$ is the skew-diffusivity associated with the scheme of Gent and McWilliams (1990) and Gent et al. (1995). $S$ is the magnitude of the neutral slope $\mathbf{S}$, which is computed via

$\mathbf{S}=-\left(\frac{\rho_{, \theta} \nabla_{z} \theta+\rho_{, s} \nabla_{z} s}{\rho_{, \theta} \theta_{, z}+\rho_{, s} s_{, z}}\right)$,

where $\rho_{, \theta}$ and $\rho_{s}$ are the partial derivatives of density with respect to potential temperature and salinity, respectively. The SGS transport tensor in Eq. (A15) results from combining the small slope neutral diffusion tensor with the skew diffusion tensor representing the Gent and McWilliams (1990) and Gent et al. (1995) eddy induced transport. Use of the combined transport tensor is not dependent on taking the same values for the diffusivities $A_{I}$ and $A_{g m}$, although this choice does result in the very simple horizontal downgradient form for the two horizontal flux components.

\section{A3 Vertically integrated budgets}

To exploit the factor of 50-100 between the speeds of fast barotropic waves and slow baroclinic waves and advection, MOM4.0 approximates the fast modes by time stepping the vertically integrated volume and momentum budgets with a small time step, whereas the slower three-dimensional dynamics are updated using longer time steps. This method and its benefits are discussed in Sect. 3.1. Here, we simply expose the equations.

The budget for volume within a column of Boussinesq seawater leads to the evolution equation for the ocean surface height $\eta$

$\eta_{, t}=-\nabla \cdot \mathbf{U}+q_{\mathrm{w}}+\mathcal{S}^{(\eta)}$.

Here,

$\mathbf{U}=\int_{-H}^{\eta} \mathrm{d} z \mathbf{u}$

is the horizontal velocity integrated from the ocean bottom at $z=-H$ to the free surface at $z=\eta, q_{\mathrm{w}}$ is the water transport across the free surface (Eq. A10), and $\mathcal{S}^{(\eta)}$ is a volume source in the column. Correspondingly, a vertical sum of the thickness weighted momentum Eq. (A1) leads to the twodimensional system

$\rho_{o}\left(\partial_{t}+f \hat{\mathbf{z}} \times\right) \mathbf{U}=-(H+\eta) \nabla_{z} p_{\mathrm{s}}+\rho_{o} \mathbf{G}$.

Here, $p_{\mathrm{s}}=\rho g \eta$ is the pressure at $z=0$ associated with mass in the region between $z=0$ and $z=\eta$, and $\mathbf{G}$ is the vertical sum of the remaining terms appearing in Eq. (A1). 


\section{A4 Dissipative aspects of the predictor-corrector}

The purpose of this section is to expose the dissipative aspects of the predictor-corrector scheme used for the barotropic equations in OM3.1. A similar treatment is given in Sect. 12.8.1 of Griffies (2004).

In two space dimensions, the predictor-corrector equations for an update of the surface height and vertically integrated horizontal velocity are

$$
\begin{aligned}
\frac{\eta^{*}-\eta^{n}}{\Delta t} & =-\gamma \nabla \cdot \mathbf{U}^{n} \\
\frac{\mathbf{U}^{n+1}-\mathbf{U}^{n}}{\Delta t} & =-c^{2} \nabla \eta^{*} \\
\frac{\eta^{n+1}-\eta^{n}}{\Delta t} & =-\nabla \cdot \mathbf{U}^{n+1},
\end{aligned}
$$

where $n$ symbolizes the barotropic time step. For brevity we dropped the fresh water and source terms appearing in Eq. (A17), and we assumed an unforced linear shallow water system with squared wave speed $c^{2}=g H$. Setting the dimensionless dissipation parameter $\gamma \geq 0$ to zero recovers a "forward-backward" scheme discussed by Killworth et al. (1991). Keeping $\gamma>0$ was useful in our simulations and was motivated by similar experiences in the Hallberg Isopycnal Model (Hallberg, 1997).

Eliminating the predicted surface height $\eta^{*}$ leads to

$$
\begin{aligned}
\frac{\mathbf{U}^{n+1}-\mathbf{U}^{n}}{\Delta t} & =-c^{2} \nabla \eta^{n}+\gamma c^{2} \Delta t \nabla\left[\nabla \cdot \mathbf{U}^{n}\right] \\
\frac{\eta^{n+1}-\eta^{n}}{\Delta t} & =-\nabla \cdot \mathbf{U}^{n+1} .
\end{aligned}
$$

To directly see how the surface height evolves, eliminate $\mathbf{U}$ to find

$$
\frac{\eta^{n+1}-2 \eta^{n}+\eta^{n-1}}{(\Delta t)^{2}}=(c \nabla)^{2} \eta^{n}+\gamma(c \nabla)^{2}\left(\eta^{n}-\eta^{n-1}\right) \text {. }
$$

Taking the limit $\Delta t \rightarrow 0$, yet with $\gamma \Delta t$ constant, leads to a dissipative wave equation

$$
\left(\partial_{t t}-c^{2} \nabla^{2}\right) \eta=(\gamma \Delta t)(c \nabla)^{2} \partial_{t} \eta .
$$

A single spatial Fourier mode with wavenumber amplitude $\kappa$ thus satisfies

$$
\left(\mathrm{d}^{2} / \mathrm{d} t^{2}+\gamma \Delta t(c \kappa)^{2} \mathrm{~d} / \mathrm{d} t+(c \kappa)^{2}\right) \eta=0 .
$$

This is the equation for a damped harmonic oscillator with inverse e-folding time $(1 / 2) \gamma \Delta t(c \kappa)^{2}$. With $\gamma>0$, external gravity waves are selectively dissipated in regions where the surface height is changing in time, and where the spatial scales are small. Faster waves are damped more readily than slower waves. These properties are useful when aiming to suppress the B-grid computational null mode discussed in Killworth et al. (1991) and Griffies et al. (2001).
Acknowledgements. This work is the result of a large number of people at GFDL over recent years. We thank in particular those in computer systems and modelling services who provided technical assistance in keeping the model running on the many different computational platforms employed during development. B. Hallberg remained throughout this development a generous source of fruitful suggestions and honest guidance. A. Adcroft, T. McDougall, M. Roberts, R. Stouffer, D. Webb, and an anonymous reviewer provided very useful critical comments which greatly improved this paper. We furthermore thank J. Mahlman and A. Leetmaa, the two directors of GFDL during the course of this project, for their patient support and encouragement.

Edited by: T. McDougall

\section{References}

Adcroft, A. and Campin, J.-M.: Rescaled height coordinates for accurate representation of free-surface flows in ocean circulation models, Ocean Modelling, 7, 269-284, 2004.

Adcroft, A., Hill, C., and Marshall, J.: Representation of topography by shaved cells in a height coordinate ocean model, Monthly Weather Review, 125, 2293-2315, 1997.

Anderson, J. L., Balaji, V., Broccoli, A. J., Cooke, W. F., Delworth, T. L., Dixon, K. W., Donnor, L. J., Dunne, K. A., Freidenreich, S. M., Garner, S. T., Gudgel, R. G., Gordon, C., Held, I. M., Hemler, R. S., Horowitz, L. W., Klein, S. A., Knuttson, T. R., Kushner, P. J., Langenhorst, A. R., Lau, N.-C., Liang, Z., Malyshev, S. L., Milly, P., Nath, M. J., Ploshay, J. J., Ramaswany, V., Schwarzkopf, M. D., Shevliakova, E., Sirutis, J. J., Soden, B. J., Stern, W. F., Thompson, L. A., Wilson, R. J., Wittenberg, A. T., and Wyman, B. L.: The GFDL Global Atmospheric Model Development Team: The new GFDL global atmosphere and land model AM2/LM2: Evaluation with prescribed SST simulations, Journal of Climate, 17, 4641-4673, 2005.

Asselin, R.: Frequency filter for time integrations, Monthly Weather Review, 100, 487-490, 1972.

Beckers, J. M., Burchard, H., Campin, J.-M., Deleersnijder, E., and Mathieu, P. P.: Another reason why simple discretizations of rotated diffusion operators cause problems in ocean models: Comments on isoneutral diffusion in a $z$-coordinate ocean model, Journal of Physical Oceanography, 28, 1552-1559, 1998.

Beckers, J. M., Burchard, H., Deleersnijder, E., and Mathieu, P. P.: Numerical discretization of rotated diffusion operators in ocean models, Monthly Weather Review, 128, 2711-2733, 2000.

Beckmann, A.: The representation of bottom boundary layer processes in numerical ocean circulation models, in: Ocean Modeling and Parameterization, edited by: Chassignet, E. P. and Verron, J., vol. 516 of NATO ASI Mathematical and Physical Sciences Series, 135-154, Kluwer, 1998.

Beckmann, A. and Döscher, R.: A method for improved representation of dense water spreading over topography in geopotentialcoordinate models, Journal of Physical Oceanography, 27, 581591, 1997.

Bentsen, M., Evensen, G., Drange, H., and Jenkins, A.: Coordinate Transformation on a Sphere Using Conformal Mapping, Monthly Weather Review, 1267, 2733-2740, 1999.

Bryan, F.: Parameter sensitivity of primitive equation ocean general circulation models, Journal of Physical Oceanography, 17, 970- 
985, 1987.

Bryan, K.: A numerical method for the study of the circulation of the world ocean, Journal of Computational Physics, 4, 347-376, 1969a.

Bryan, K.: Climate and the ocean circulation III: The ocean model, Monthly Weather Review, 97, 806-824, 1969b.

Bryan, K. and Cox, M. D.: A numerical investigation of the oceanic general circulation, Tellus, XIX, 54-80, 1967.

Bryan, K. and Cox, M. D.: An approximate equation of state for numerical models of the ocean circulation, Journal of Physical Oceanography, 4, 510-514, 1972.

Bryan, K. and Lewis, L. J.: A water mass model of the world ocean, Journal of Geophysical Research, 84, 2503-2517, 1979.

Bryan, K., Manabe, S., and Pacanowski, R. C.: A global oceanatmosphere climate model. Part II. The oceanic circulation, Journal of Physical Oceanography, 5, 30-46, 1975.

Bryan, K., Dukowicz, J. K., and Smith, R. D.: On the Mixing Coefficient in the Parameterization of Bolus Velocity, Journal of Physical Oceanography, 29, 2442-2456, 1999.

Campin, J.-M., Adcroft, A., Hill, C., and Marshall, J.: Conservation of properties in a free-surface model, Ocean Modelling, 6, 221244, 2004

Conkright, M., Antonov, J., Baranova, O., Boyer, T., Garcia, H., Gelfeld, F., Johnson, D., Locarnini, R., Murphy, P., O'Brien, T., Smolyar, I., and Stephens, C.: World Ocean Database 2001, Volume 1: Introduction, NOAA Atlas NESD' is 42, US Government Printing Office 13, NOAA, Washington, D.C., 167 pp., 2002.

Coward, A., Killworth, P., and Blundell, J.: Tests of a twogrid world ocean model, Journal of Geophysical Research, 99, 22 725-22 735, 1994.

Cox, M. D.: A Primitive Equation, 3-Dimensional Model of the Ocean, NOAA/Geophysical Fluid Dynamics Laboratory, Princeton, USA, 1984.

Cox, M. D.: Isopycnal diffusion in a $z$-coordinate ocean model, Ocean Modelling, 74, 1-5, 1987.

Cunningham, S., Alderson, S., King, B., and Brandon, M.: Transport and variability of the Antarctic Circumpolar Current in Drake Passage, Journal of Geophysical Research, 108, Art. 8084, 2003.

Danabasoglu, G., McWilliams, J. C., and Gent, P.: The role of mesoscale tracer transports in the global ocean circulation, Science, 8, 1123-1126, 1994.

Danabasoglu, G., Large, W., Tribbia, J., Gent, P., Briegleb, B., and McWilliams, J. C.: Diurnal ocean-atmosphere coupling, Journal of Climate, accepted, 2005.

Deleersnijder, E., Van Ypersele, J.-P., and Campin, J.-M.: An orthogonal curvilinear coordinate system for a world ocean model, Ocean Modelling, 100, 7-10, 1993.

Delworth, T. L., Stouffer, R., Dixon, K., Spelman, M., Knutson, T., Broccoli, A., Kushner, P., and Wetherald, R.: Review of simulations of climate variability and change with the GFDL R30 coupled climate model, Climate Dynamics, 19, 555-574, 2002.

Delworth, T. L., Broccoli, A. J., Rosati, A., Stouffer, R. J., Balaji, V., Beesley, J. A., Cooke, W. F., Dixon, K. W., Dunne, J., Dunne, K. A., Durachta, J. W., Findell, K. L., Ginoux, P., Gnanadesikan, A., Gordon, C., Griffies, S. M., Gudgel, R., Harrison, M. J., Held, I. M., Hemler, R. S., Horowitz, L. W., Klein, S. A., Knutson, T. R., Kushner, P. J., Langenhorst, A. L., Lee, H.-C., Lin, S., Lu, L., Malyshev, S. L., Milly, P., Ramaswamy, V., Russell, J.,
Schwarzkopf, M. D., Shevliakova, E., Sirutis, J., Spelman, M., Stern, W. F., Winton, M., Wittenberg, A. T., Wyman, B., Zeng, F., and Zhang, R.: GFDL's CM2 Global Coupled Climate Models - Part 1: Formulation and Simulation Characteristics, Journal of Climate, accepted, 2005.

Dewar, W. K., Hsueh, Y., McDougall, T. J., and Yuan, D.: Calculation of Pressure in Ocean Simulations, Journal of Physical Oceanography, 28, 577-588, 1998.

Doney, S. C. and Hecht, M. W.: Antarctic Bottom Water formation and deep water chlorofluorocarbon distributions in a global ocean climate model, Journal of Physical Oceanography, 32, 1642-1666, 2002.

Döscher, R. and Beckmann, A.: Effects of a bottom boundary layer parameterization in a coarse-resolution model of the North Atlantic Ocean, Journal of Atmospheric and Oceanic Technology, 17, 698-707, 2000.

Dukowicz, J. K. and Smith, R. D.: Stochastic theory of compressible turbulent fluid transport, Physics of Fluids, 9, 3523-3529, 1997.

Durran, D. R.: Numerical Methods for Wave Equations in Geophysical Fluid Dynamics, Springer Verlag, Berlin, 470 pp., 1999.

Eby, M. and Holloway, G.: Grid transformation for incorporating the Arctic in a global ocean model, Climate Dynamics, 10, 241247, 1994.

Egbert, G., Bennett, A., and Foreman, M.: TOPEX/POSEIDON tides estimated using inverse model, Journal of Geophysical Research, 99, 24 821-24 852, 1994.

Feistel, R. and Hagen, E.: On the Gibbs thermodynamic potential of seawater., Progress in Oceanography, 36, 249-327, 1995.

Ferrari, R. and Plumb, A. R.: Residual circulation in the ocean, in: Near-Boundary Processes and Their Parameterization, edited by: Müller, P. and Garrett, C., Proceedings of the 13th 'Aha Huliko'a Hawaiian Winter Workshop, University of Hawaii at Manoa, 219-228, 2003.

Gent, P. R. and McWilliams, J. C.: Isopycnal mixing in ocean circulation models., Journal of Physical Oceanography, 20, 150-155, 1990.

Gent, P. R., Willebrand, J., McDougall, T. J., and McWilliams, J. C.: Parameterizing eddy-induced tracer transports in ocean circulation models, Journal of Physical Oceanography, 25, 463-474, 1995.

Gerdes, R., Köberle, C., and Willebrand, J.: The influence of numerical advection schemes on the results of ocean general circulation models, Climate Dynamics, 5, 211-226, 1991.

Gerdes, R., Hurlin, W., and Griffies, S.: Sensitivity of a global ocean model to increased run-off from Greenland, Ocean Modelling, accepted, 2005.

Gill, A.: Atmosphere-Ocean Dynamics, vol. 30 of International Geophysics Series, Academic Press, London, 662 + xv pp., 1982.

Gnanadesikan, A.: A simple predictive model for the structure of the oceanic pycnocline, Science, 283, 2077-2079, 1999a.

Gnanadesikan, A.: A global model of silicon cycling: Sensitivity to eddy parameterization and dissolution, Global Biogeochemical Cycles, 13, 199-220, 1999b.

Gnanadesikan, A.: Numerical issues for coupling biological models with isopycnal mixing schemes, Ocean Modelling, 1, 1-15, 1999c.

Gnanadesikan, A., Slater, R., Gruber, N., and Sarmiento, J.: Oceanic vertical exchange and new production: A comparison 
between models and data, Deep Sea Research II, 49, 363-401, 2002.

Gnanadesikan, A., Slater, R., and Samuels, B.: Sensitivity of water mass transformation and heat transport to subgridscale parameterization in ocean general circulation models, Geophysical Research Letters, 30, 1967, doi:10.1029/2003GL018 036, 2003.

Gnanadesikan, A., Dixon, K. W., Griffies, S. M., Balaji, V., Beesley, J. A., Cooke, W. F., Delworth, T. L., Gerdes, R., Harrison, M. J., Held, I. M., Hurlin, W. J., Lee, H.-C., Liang, Z., Nong, G., Pacanowski, R. C., Rosati, A., Russell, J., Samuels, B. L., Song, S. M., , Spelman, M. J., Stouffer, R. J., Sweeney, C. O., Vecchi, G., Winton, M., Wittenberg, A. T., Zeng, F., and Zhang, R.: GFDL's CM2 Global Coupled Climate Models-Part 2: The Baseline Ocean Simulation, Journal of Climate, accepted, 2005a.

Gordon, A., Susanto, R., and Vranes, K.: Cool Indonesian throughflow as a consequence of restricted surface layer flow, Nature, $425,824-828,2003$.

Gordon, C., Cooper, C., Senior, C. A., Banks, H., J. M. Gregory, T. C. J., Mitchell, J. F. B., and Wood, R. A.: The simulation of SST, sea ice extents and ocean heat transports in a version of the Hadley Centre coupled model without flux adjustments., Climate Dynamics, 16, 147-168, 2000.

Greatbatch, R. J. and Li, G.: Alongslope mean flow and an associated upslope bolus flux of tracer in a parameterization of mesoscale turbulence, Deep-Sea Research, 47, 709-735, 2000.

Gregg, M., Sanford, T., and Winkel, D.: Reduced mixing from the breaking of internal waves in equatorial waters, Nature, 422 , 513-515, 2003

Griffies, S. M.: The Gent-McWilliams skew-flux, Journal of Physical Oceanography, 28, 831-841, 1998.

Griffies, S. M.: Fundamentals of ocean climate models, Princeton University Press, Princeton, USA, 518+xxxiv pages, 2004.

Griffies, S. M. and Hallberg, R. W.: Biharmonic friction with a Smagorinsky viscosity for use in large-scale eddy-permitting ocean models, Monthly Weather Review, 128, 2935-2946, 2000.

Griffies, S. M., Gnanadesikan, A., Pacanowski, R. C., Larichev, V., Dukowicz, J. K., and Smith, R. D.: Isoneutral diffusion in a $z$ coordinate ocean model, Journal of Physical Oceanography, 28, 805-830, 1998.

Griffies, S. M., Böning, C., Bryan, F. O., Chassignet, E. P., Gerdes, R., Hasumi, H., Hirst, A., Treguier, A.-M., and Webb, D.: Developments in Ocean Climate Modelling, Ocean Modelling, 2, 123-192, 2000a.

Griffies, S. M., Pacanowski, R. C., and Hallberg, R. W.: Spurious diapycnal mixing associated with advection in a $z$-coordinate ocean model, Monthly Weather Review, 128, 538-564, $2000 \mathrm{~b}$.

Griffies, S. M., Pacanowski, R., Schmidt, R., and Balaji, V.: Tracer Conservation with an Explicit Free Surface Method for $z$ coordinate Ocean Models, Monthly Weather Review, 129, 10811098, 2001

Griffies, S. M., Harrison, M. J., Pacanowski, R. C., and Rosati, A.: A Technical Guide to MOM4, NOAA/Geophysical Fluid Dynamics Laboratory, Princeton, USA, 337 pp., 2004.

Hallberg, R. and Gnanadesikan, A.: An exploration of the role of transient eddies in determining the transport of a zonally reentrant current, Journal of Physical Oceanography, 31, 3312-3330, 2001.

Hallberg, R. W.: Stable split time stepping schemes for large-scale ocean modeling, Journal of Computational Physics, 135, 54-65,
1997.

Haltiner, G. T. and Williams, R. T.: Numerical Prediction and Dynamic Meteorology, John Wiley and Sons, New York, USA, 1980.

Held, I. M. and Larichev, V. D.: A scaling theory for horizontally homogeneous baroclinically unstable flow on a beta plane, Journal of Atmospheric Sciences, 53, 946-952, 1996.

Held, I. M. and Schneider, T.: The surface branch of the zonally averaged mass transport circulation in the troposphere, Journal of Atmospheric Sciences, 56, 1688-1697, 1999.

Holland, W. R., Chow, J. C., and Bryan, F. O.: Application of a third-order upwind scheme in the NCAR ocean model, Journal of Climate, 11, 1487-1493, 1998.

Huang, R. X.: Real freshwater flux as a natural boundary condition for the salinity balance and thermohaline circulation forced by evaporation and precipitation, Journal of Physical Oceanography, 23, 2428-2446, 1993.

Hundsdorfer, W. and Trompert, R.: Method of lines and direct discretization: a comparison for linear advection, Applied Numerical Mathematics, 469-490, 1994.

Jakobssen, M., Chervis, N., Woodward, J., Macnab, R., and Coakley, B.: New grid of Arctic bathymetry aids scientists and mapmakers, EOS Transactions of the American Geophysical Union, $81,89,93,96,2000$.

Kantha, L. H. and Clayson, C. A.: Small Scale Processes in Geophysical Fluid Flows, Academic Press, New York, USA, 883 pp., 2000.

Karoly, D., McIntosh, P., Berrisford, P., McDougall, T. J., and Hirst, A.: Similarities of the Deacon cell in the Southern Ocean and Ferrel cells in the atmosphere, Quarterly Journal of the Royal Meteorological Society, 123, 519-526, 1997.

Killworth, P. D.: Topographic instabilities in level model OGCM's, Ocean Modelling, 75, 9-12, 1987.

Killworth, P. D., Stainforth, D., Webb, D. J., and Paterson, S. M.: The development of a free-surface Bryan-Cox-Semtner ocean mode, Journal of Physical Oceanography, 21, 1333-1348, 1991.

Large, W. G., McWilliams, J. C., and Doney, S. C.: Oceanic vertical mixing: A review and a model with a nonlocal boundary layer parameterization, Reviews of Geophysics, 32, 363-403, 1994.

Large, W. G., Danabasoglu, G., Doney, S. C., and McWilliams, J. C.: Sensitivity to surface forcing and boundary layer mixing in a global ocean model: annual-mean climatology, Journal of Physical Oceanography, 27, 2418-2447, 1997.

Large, W. G., Danabasoglu, G., McWilliams, J. C., Gent, P. R., and Bryan, F. O.: Equatorial circulation of a global ocean climate model with anisotropic horizontal viscosity, Journal of Physical Oceanography, 31, 518-536, 2001.

Latif, M., Anderson, D., Barnett, T., Cane, M., Kleeman, R., Leetmaa, A., O'Brien, J., Rosati, A., and Schneider, E.: A review of the predictability and prediction of ENSO, Journal of Geophysical Research, 103, 14 375-14 393, 1998.

Laurent, L. C. S. and Schmitt, R.: The contribution of salt fingers to vertical mixing in the North Atlantic tracer release experiment, Journal of Physical Oceanography, 29, 1404-1424, 1999.

Leaman, K., Molinari, R., and Vertes, P.: Structure and variability of the Florida Current at 27N: April 1982-July 1984, Journal of Physical Oceanography, 17, 565-583, 1987.

Ledwell, J. R., Watson, A. J., and Law, C. S.: Evidence for slow mixing across the pycnocline from an open-ocean tracer-release 
experiment, Nature, 364, 701-703, 1993.

Lee, H.-C., Rosati, A., and Spelman, M.: Barotropic tidal mixing effects in a coupled climate model: Oceanic conditions in the northern Atlantic, Ocean Modelling, accepted, 2005.

Leonard, B. P.: A stable and accurate convective modelling procedure based on quadratic upstream interpolation, Computer Methods in Applied Mechanics and Engineering, 19, 59-98, 1979.

Lin, S.-J.: A vertically lagrangian finite-volume dynamical core for global models, Monthly Weather Review, 2293-2307, 2004.

Madec, G. and Imbard, M.: A global ocean mesh to overcome the North Pole singularity, CD, 12, 381-388, 1996.

Maier-Reimer, E., Hasselmann, K., Olbers, D., and Willebrand, J.: An ocean circulation model for climate studies, Max-PlankInstitut für Meteorologie, Hamburg, Germany, 34 pp., 1983.

Manabe, S. and Bryan, K.: Climate calculations with a combined ocean-atmosphere model, Journal of Atmospheric Sciences, 26, 786-789, 1969.

Marotzke, J.: Influence of convective adjustment on the stability of the thermohaline circulation, Journal of Physical Oceanography, 21, 903-907, 1991

Marshall, J., Olbers, D., Ross, H., and Wolf-Gladrow, D.: Potential vorticity constraints on the dynamics and hydrography of the Southern Ocean, Journal of Physical Oceanography, 23, 465487, 1993

Marshall, J., Hill, C., Perelman, L., and Adcroft, A.: Hydrostatic, quasi-hydrostatic, and nonhydrostatic ocean modeling, Journal of Geophysical Research, 102, 5733-5752, 1997.

Marsland, S., Haak, H., Jungclaus, J., Latif, M., and Röske, F.: The Max-Planck-Institute global ocean/sea ice model with orthogonal curvilinear coordinates, Ocean Modelling, 5, 91-127, 2003.

McDougall, T. J.: Neutral surfaces, Journal of Physical Oceanography, 17, 1950-1967, 1987.

McDougall, T. J. and Church, J. A.: Pitfalls with numerical representations of isopycnal and diapycnal mixing, Journal of Physical Oceanography, 16, 196-199, 1986.

McDougall, T. J. and McIntosh, P. C.: The temporal-residual-mean velocity. Part II: isopycnal interpretation and the tracer and momentum equations, Journal of Physical Oceanography, 31, 12221246, 2001.

McDougall, T. J., Jackett, D. R., Wright, D. G., and Feistel, R.: Accurate and computationally efficient algorithms for potential temperature and density of seawater, Journal of Atmospheric and Oceanic Technology, 20, 730-741, 2003.

Meehl, G., Gent, P. R., Arblaster, J., Otto-Bliesner, B., Brady, E., and Craig, A.: Factors that affect the amplitude of El Niño in global coupled climate models, Climate Dynamics, 17, 515-526, 2001

Mesinger, F. and Arakawa, A.: Numerical Methods used in Atmospheric Models, in: GARP Publication Series, 1, 66 pp., 1976.

Morel, A. and Antoine, D.: Heating rate within the upper ccean in relation to its bio-optical state, Journal of Physical Oceanography, 24, 1652-1665, 1994.

Murray, R. J.: Explicit generation of orthogonal grids for ocean models, Journal of Computational Physics, 126, 251-273, 1996.

Murray, R. J. and Reason, C. J. C.: A curvilinear version of the Bryan-Cox ocean model, Journal of Computational Physics, 171, $1-46,2002$.

Murtugudde, R., Beauchamp, J., McClain, C. R., Lewis, M., and Busalacchi, A. J.: Effects of penetrative radiation on the up- per tropical ocean circulation, Journal of Climate, 15, 470-486, 2002.

NOAA: Data Announcement 88-MGG-02, Digital relief of the Surface of the Earth, Tech. rep., NOAA, National Geophysical Data Center, Boulder, Colorado, 1988.

Ohlmann, J.: Ocean Radiant Heating in Climate Models, JOC, 16, 1337-1351, 2003.

Olbers, D. J., Wenzel, M., and Willebrand, J.: The inference of North Atlantic circulation patterns from climatological hydrographic data, Reviews of Geophysics, 23, 313-356, 1985.

Pacanowski, R. C.: Effect of equatorial currents on surface stress, Journal of Physical Oceanography, 17, 833-838, 1987.

Pacanowski, R. C. and Gnanadesikan, A.: Transient response in a $z$-level ocean model that resolves topography with partial-cells, Monthly Weather Review, 126, 3248-3270, 1998.

Pacanowski, R. C. and Griffies, S. M.: The MOM3 Manual, NOAA/Geophysical Fluid Dynamics Laboratory, Princeton, USA, $680 \mathrm{pp}, 1999$.

Park, Y.-G. and Bryan, K.: Comparison of thermally driven circulations from a depth-coordinate model and an isopycnal-layer model. Part I: scaling-law sensitivity to vertical diffusivity, Journal of Physical Oceanography, 30, 590-605, 2000.

Polzin, K. L., Speer, K., Toole, J. M., and Schmitt, R. W.: Intense mixing of Antarctic bottom water in the equatorial Atlantic, Nature, 380, 54-57, 1996.

Polzin, K. L., Toole, J. M., Ledwell, J. R., and Schmitt, R. W.: Spatial variability of turbulent mixing in the abyssal ocean, Science, 276, 93-96, 1997.

Rahmstorf, S.: A fast and complete convection scheme for ocean models, Ocean Modelling, 101, 9-11, 1993.

Redi, M. H.: Oceanic isopycnal mixing by coordinate rotation, Journal of Physical Oceanography, 12, 1154-1158, 1982.

Roach, A., Aagard, K., Pease, C., Salo, S., Weingartner, T., Pavlov, V., and Kulakov, M.: Direct measurements of transport and water properties through Bering Strait, Journal of Geophysical Research, 100, 18 443-18 457, 1995.

Robert, A.: The integration of a low order spectral form of the primitive meteorological equations, Journal of the Meteorological Society of Japan, 44, 237-244, 1966.

Roberts, J., Heil, P., Murray, R., Holloway, D., and Bindoff, N.: Pole relocation for an orthogonal grid: an analytic method, Ocean Modelling, accepted, 2005.

Roberts, M. J. and Marshall, D.: Do we require adiabatic dissipation schemes in eddy-resolving ocean models?, Journal of Physical Oceanography, 28, 2050-2063, 1998.

Roberts, M. J. and Wood, R.: Topographic sensitivity studies with a Bryan-Cox-type ocean model, Journal of Physical Oceanography, 27, 823-836, 1997.

Schmitt, R. W.: Double diffusion in oceanography, Annual Review of Fluid Mechanics, 26, 255-285, 1994.

Schneider, E., DeWitt, D., Rosati, A., Kirtman, B., Link, J., and Tribbia, J.: Retrospective ENSO forecasts: sensitivity to atmospheric model and ocean resolution, Monthly Weather Review, 131, 3038-3060, 2003.

Semtner, A. J.: An oceanic general circulation model with bottom topography, in Numerical Simulation of Weather and Climate, Technical Report No. 9, UCLA Department of Meteorology, 1974.

Simmons, H. L., Jayne, S. R., Laurent, L. C. S., and Weaver, A. J.: 
Tidally driven mixing in a numerical model of the ocean general circulation, Ocean Modelling, 6, 245-263, 2004.

Smagorinsky, J.: General circulation experiments with the primitive equations: I. The basic experiment, Monthly Weather Review, 91, 99-164, 1963.

Smagorinsky, J.: Some historical remarks on the use of nonlinear viscosities, in: Large Eddy Simulation of Complex Engineering and Geophysical Flows, edited by: Galperin, B. and Orszag, S. A., Cambridge University Press, 3-36, 1993.

Smith, K. S. and Vallis, G. K.: The scales and equilibration of midocean eddies: forced-dissipative flow, Journal of Physical Oceanography, 32, 1699-1721, 2002.

Smith, R. D. and McWilliams, J. C.: Anisotropic horizonal viscosity for ocean models, Ocean Modelling, 5, 129-156, 2003.

Smith, R. D., Kortas, S., and Meltz, B.: Curvilinear coordinates for global ocean models, Los Alamos preprint, LA-UR-95-1146, 1995.

Smith, W. H. F. and Sandwell, D. T.: Global seafloor topography from satellite altimetry and ship depth soundings, Science, 277, 1957-1962, 1997.

Solomon, H.: On the representation of isentropic mixing in ocean models, Journal of Physical Oceanography, 1, 233-234, 1971.

Stacey, M. W., Pond, S., and Nowak, Z. P.: A Numerical Model of the Circulation in Knight Inlet, British Columbia, Canada, Journal of Physical Oceanography, 25, 1037-1062, 1995.

Stammer, D.: Global characteristics of ocean variability estimated from regional TOPEX/POSEIDON altimeter measurements, Journal of Physical Oceanography, 27, 1743-1769, 1997.

Stone, P.: A simplified radiative-dynamical model for the static stability of rotating atmospheres, Journal of Atmospheric Sciences, 29, 405-418, 1972.

Stouffer, R. J., Broccoli, A., Delworth, T., Dixon, K., Gudgel, R., Held, I., Hemler, R., Knutsn, T., Lee, H.-C., Schwarzkopf, M., Soden, B., Spelman, M., Winton, M., and Zeng, F.: GFDL's CM2 Global coupled climate models: Part 4: idealized climate response, Journal of Climate, accepted, 2005.

Sweby, P.: High-resolution schemes using flux limiters for hyperbolic conservation-laws, SIAM Journal of Numerical Analysis, 21, 995-1011, 1984.
Sweeney, C., Gnanadesikan, A., Griffies, S. M., Harrison, M., Rosati, A., and Samuels, B.: Impacts of shortwave penetration depth on large-scale ocean circulation and heat transport, Journal of Physical Oceanography, 35, 1103-1119, 2005.

Tang, Y. and Roberts, M.: The impact of a bottom boundary layer scheme on the North Atlantic Ocean in a global coupled climate model, Journal of Physical Oceanography, 35, 202-217, 2005.

Toole, J. M. and McDougall, T. J.: Mixing and stirring in the ocean interior, in: Ocean Circulation and Climate: Observing and Modelling the Global Ocean, edited by: Siedler, G., Gould, J., and Church, J., Academic Press, 337-356, 2001.

Toole, J. M., Polzin, K. L., and Schmitt, R. W.: Estimates of diapycnal mixing in the abyssal ocean, Science, 264, 1120-1123, 1994.

Toole, J. M., Schmitt, R. W., and Polzin, K. L.: Near-boundary mixing above the flanks of a mid-latitude seamount, Journal of Geophysical Research, 102, 947-959, 1997.

Treguier, A. M., Held, I. M., and Larichev, V. D.: On the parameterization of quasi-geostrophic eddies in primitive equation ocean models, Journal of Physical Oceanography, 27, 567-580, 1997.

Visbeck, M., Marshall, J. C., Haine, T., and Spall, M.: Specification of eddy transfer coefficients in coarse resolution ocean circulation models, Journal of Physical Oceanography, 27, 381-402, 1997.

Wajsowicz, R. C.: Models of the Southeast Asian Seas, Journal of Physical Oceanography, 29, 986-1018, 1999.

Whitehead, J. and Worthington, L.: The flux and mixing rates of Antarctic Bottom Water within the North Atlantic, Journal of Geophysical Research, 87, 7903-7924, 1982.

Winton, M., Hallberg, R. W., and Gnanadesikan, A.: Simulation of density-driven frictional downslope flow in $z$-coordinate ocean models, Journal of Physical Oceanography, 28, 2163 2174, 1998.

Wittenberg, A., Rosati, A., Lau, G., and Ploshay, J.: Tropical simulations with the recent GFDL coupled climate model, Journal of Climate, accepted, 2005. 